\title{
On the reliability of mass-loss-rate estimates for AGB stars ${ }^{\star}$
}

\author{
S. Ramstedt ${ }^{1}$, F. L. Schöier ${ }^{1,2}$, H. Olofsson ${ }^{1,2}$, and A. A. Lundgren ${ }^{3}$ \\ ${ }^{1}$ Stockholm Observatory, AlbaNova University Center, 10691 Stockholm, Sweden \\ e-mail: sofia@astro.su.se \\ 2 Onsala Space Observatory, 43992 Onsala, Sweden \\ 3 European Southern Observatory, Casilla 19001, Santiago 19, Chile \\ Received 19 October 2007 / Accepted 30 May 2008
}

\section{ABSTRACT}

\begin{abstract}
Context. In the recent literature there has been some doubt as to the reliability of CO multi-transitional line observations as a massloss-rate estimator for AGB stars.

Aims. Using new well-calibrated CO radio line observations, the main aim of the work presented here is to carefully evaluate the reliability of CO mass-loss-rate estimates for intermediate- to high-mass-loss-rate AGB stars with different photospheric chemistries. Methods. Mass-loss rates for 10 intermediate- to high-mass-loss-rate AGB stars are derived using a detailed non-LTE, non-local radiative transfer code based on the Monte-Carlo method to model the $\mathrm{CO}$ radio line intensities. The circumstellar envelopes are assumed to be spherically symmetric and formed by constant mass-loss rates. The energy balance is solved self-consistently and the effects of dust on the radiation field and thermal balance included. An independent estimate of the mass-loss rate is also obtained from the combination of dust radiative transfer modelling with a dynamical model of the gas and dust particles.

Results. We find that the $\mathrm{CO}$ radio line intensities and shapes are successfully reproduced for the majority of our objects when assuming a constant mass-loss rate. Moreover, the CO line intensities are only weakly dependent on the adopted micro-turbulent velocity, in contrast to recent claims in the literature. The two methods used in the present work to derive mass-loss rates are consistent within a factor of $\sim 3$ for intermediate- to high-mass-loss-rate objects, indicating that this is a lower limit to the uncertainty in present mass-loss-rate estimates. We find a tentative trend with chemistry. Mass-loss rates from the dust/dynamical model are systematically higher than those from the $\mathrm{CO}$ model for the carbon stars and vice versa for the M-type stars. This could be ascribed to a discrepancy in the adopted $\mathrm{CO} / \mathrm{H}_{2}$-abundance ratio, but we caution that the sample is small and systematic errors cannot be excluded.
\end{abstract}

Key words. stars: AGB and post-AGB - stars: carbon - stars: late-type - stars: mass-loss

\section{Introduction}

Mass loss is the single most important process during the final evolution of low- and intermediate-mass stars on the asymptotic giant branch (AGB) (Bloecker 1995). The mass loss determines the lifetime on the AGB and thus the maximum luminosity and radius reached (e.g., Marigo \& Girardi 2007). Furthermore, since AGB stars are major contributors of mass to the interstellar medium (Sedlmayr 1994; Dorschner \& Henning 1995) and to the integral luminosity of intermediate-age stellar systems, an understanding of the mass-loss phenomenon is also important for studying extra-galactic populations (Battinelli \& Demers 2004) and for the chemical evolution of galaxies (Schröder \& Sedlmayr 2001; see also Karakas \& Lattanzio 2007, for a recent paper on yields from AGB stars). The existence of mass loss is well established (Willson 2000), but its details are less well known. This is unfortunate since even a modest mass-loss-rate change, e.g. by a factor of two, will have a profound effect on the evolution of the star, its nucleosynthesis, and its mass return to the interstellar medium (Forestini \& Charbonnel 1997).

Although mass loss on the AGB can reach rates as high as $10^{-4} M_{\odot} \mathrm{yr}^{-1}$, the wind speed is quite low $\left(\leq 30 \mathrm{~km} \mathrm{~s}^{-1}\right)$. The temperature of the outer parts of the atmosphere is low enough for dust to condense. These facts, combined with a high stellar luminosity $\left(\sim 10^{4} L_{\odot}\right)$, have lead to the conclusion that the winds of AGB stars are, at least partly, dust-driven, i.e., the mass loss from the star is driven by radiation pressure on grains and the gas

* Appendices A-C are only available in electronic form at http://www . aanda.org is dragged away from the star by momentum-coupling between the grains and the gas (e.g., Lamers \& Cassinelli 1999). The effects of the gas-dust interaction on the mass loss and wind formation have been studied by several authors (e.g., Habing 1996; Simis et al. 2001). Time-dependent dynamical models studying the effects of stellar pulsations (Bowen 1988) and further including detailed time-dependent dust formation (Höfner et al. 1995) have progressed to dynamical models in which the motion of the gas and dust particles is calculated and the radiative transfer is solved (Sandin \& Höfner 2003). These models work well for carbon stars and support the dust-driven wind scenario, but the formation of silicate grains close enough to the star to drive mass-loss rates of the observed magnitude still poses a problem for M-type stars (Woitke 2006; Höfner \& Andersen 2007).

Most likely, the average mass-loss rate increases as the star evolves along the AGB (Habing 1996; Schöier \& Olofsson 2001). In addition to this steady increase, modulations of the mass-loss rate on shorter time scales are present (Habing 1996). Indications of this can be seen in observations of circumstellar CO line emission around some AGB stars with doublecomponent line profiles (Knapp et al. 1998; Winters et al. 2003) and in observations of stellar and ambient Galactic light scattered by dust in circumstellar envelopes (CSEs) (Mauron \& Huggins 2000, 2001, 2006). A process which can have a strong effect on the mass-loss properties of an AGB-star is the He-shell flash (or thermal pulse) (Olofsson et al. 1993, 2000; Steffen \& Schönberner 2000; Schöier et al. 2005a). Other processes may be important during the very last stages when the star sheds the remaining few tenths of a solar mass of its hydrogen envelope 
(during a few hundred years) before it leaves the AGB (Skinner et al. 1997). Finally, the presence of a binary companion may influence the magnitude as well as the characteristics (e.g., the geometry) of the mass loss.

To make progress in the study of AGB stars, it is important to establish reliable mass-loss-rate estimators, which can be used primarily over a wide range of mass-loss rates and time scales and for different chemical types (M-, S-, and C-stars). Estimating AGB mass-loss rates through observations of circumstellar CO radio line emission, in combination with modelling of the radiative transfer, is considered to be one of the most reliable methods. Carbon monoxide line emission has also been used in a number of studies aimed at tracing the mass-loss history (Schöier et al. 2002; Kemper et al. 2003; Teyssier et al. 2006; Decin et al. 2007) and at identifying drastic mass-loss-rate changes at the end of the AGB (Heske et al. 1990; Justtanont et al. 1996). Detailed studies of the mass-loss properties of carbon stars (Schöier \& Olofsson 2001; Schöier et al. 2002), M-type AGB stars (Olofsson et al. 2002; González Delgado et al. 2003), and S-type AGB stars (Ramstedt et al. 2006) have been performed on samples dominated by low-mass-loss-rate objects. It was concluded that for these types of objects the $\mathrm{CO}$ radio line emission is a good mass-loss-rate estimator, although not entirely free of problems.

It has been known for some time that for the higher massloss-rate objects the situation is less clear (Sahai 1990; Kastner 1992; Schöier \& Olofsson 2001). A higher mass-loss rate leads to a decrease in the drift velocity between the dust and the gas and therefore a less efficient heating of the gas through collisions with dust grains. Since CO line emission is also a dominant coolant, the effect of an increased mass-loss rate is increased cooling of the circumstellar gas. This, in combination with saturation of the spectral lines, leads to a situation where the integrated CO line intensities are less dependent on the mass-loss rate when it exceeds about $10^{-5} M_{\odot} \mathrm{yr}^{-1}$; the higher the frequency of the lines the lower the mass-loss rate when the effect sets in. This makes CO line emission a questionable massloss-rate estimator at mass-loss rates in excess of $10^{-5} M_{\odot} \mathrm{yr}^{-1}$, an unfortunate effect since we know that mass-loss rates up to $10^{-4} M_{\odot} \mathrm{yr}^{-1}$ are reached during the very final stage, and this is also when the stars make their largest contribution to the return of nuclear-processed material to the interstellar medium. In addition, Kemper et al. (2003) concluded that standard circumstellar radiative transfer models could not explain some CO line intensity ratios, and that the mass-loss-rate estimate is sensitively dependent on the (unknown) local turbulent line width.

An alternative way to estimate the mass-loss rate, not subject to the same problems, is to combine modelling of the dust radiative transfer with solving the coupled equations of motion for the gas and the dust particles (Habing et al. 1994). The gas expansion velocity is a strong function of the dust-to-gas mass-lossrate ratio and therefore a solution to the kinematical equations that fits the gas expansion velocity and the dust optical depth, obtained through dust radiative transfer modelling, results in an independent estimate of the mass-loss rate.

Presented here are a detailed radiative transfer modelling of primarily new, well-calibrated, and high signal-to-noise ratio observations of $\mathrm{CO} J=1 \rightarrow 0,2 \rightarrow 1,3 \rightarrow 2,4 \rightarrow 3$, and $6 \rightarrow 5$ emission from a sample of 10 well-studied high-mass-loss-rate AGB stars and the resulting mass-loss-rate estimates. In addition, dust emission modelling combined with a dynamical model give independent estimates of the mass-loss rates. The dust emission modelling also provides the dust information necessary for the $\mathrm{CO}$ modelling, e.g., the dust radiation field. By obtaining
Table 1. The sample with spectral types, variable types, periods $(P)$, luminosities $\left(L_{\star}\right)$, and distances $(D)$.

\begin{tabular}{lccccr}
\hline \hline Source & $\begin{array}{c}\text { Spec. } \\
\text { Type }\end{array}$ & Type & $\begin{array}{c}P \\
{[\text { days }]}\end{array}$ & $\begin{array}{c}L_{\star} \\
{\left[L_{\odot}\right]}\end{array}$ & $\begin{array}{c}D \\
{[\mathrm{pc}]}\end{array}$ \\
\hline LP And & C & Mira & 620 & 8200 & 620 \\
CW Leo & C & Mira & 630 & 8300 & 120 \\
RW LMi & C & SRa & 640 & 8500 & 390 \\
V384 Per & C & Mira & 535 & 7000 & 590 \\
AFGL 3068 & C & Mira & 696 & 9300 & 1090 \\
& & & & & \\
TX Cam & M & Mira & 557 & 11900 & 440 \\
GX Mon & M & Mira & 527 & 11100 & 630 \\
WX Psc & M & Mira & 660 & 14600 & 720 \\
IK Tau & M & Mira & 500 & 10400 & 300 \\
IRC-10529 & M & Mira & 680 & 15100 & 890 \\
\hline
\end{tabular}

the mass-loss rates for the stars using two independent methods, we can evaluate the reliability of the mass-loss-rate estimators and also look for trends with e.g. mass-loss rate and chemistry. A similar investigation comparing mass-loss-rates derived from ISO-SWS observations of the silicate dust features with massloss rates from $\mathrm{CO}$ modelling found in the literature on a sample of low-mass-loss-rate M-type AGB stars has been performed by Heras \& Hony (2005).

The sample and the observational data are presented in Sects. 2 and 3. In Sect. 4 the adopted circumstellar model is discussed. In Sect. 5 the CO radiative transfer model is described and $\mathrm{CO}$ as a mass-loss-rate estimator is discussed. In Sect. 6, we describe how dust radiative transfer and the dynamical model is used to estimate mass-loss rates. The results are presented in Sect. 7 and outlined for each individual object in Sect. 8. In Sect. 9 we discuss and compare the two methods, and in Sect. 10 we give our conclusions.

\section{The sample}

The sample, presented in Table 1, consists of 10 well-studied AGB stars, with intermediate to high mass-loss rates. They are chosen with a spread in mass-loss rate, to allow studies of possible trends. Dependences on chemistry can also be investigated, since half of the stars were chosen to have a photospheric $\mathrm{C} / \mathrm{O}$ ratio $>1$ (carbon stars) and the other half $\mathrm{C} / \mathrm{O}<1$ (M-type).

Bolometric luminosities are estimated using the periodluminosity relation of Feast et al. (2006) for the carbon stars, and that of Whitelock et al. (1994) for the M-type stars. Periods are mainly taken from the Combined General Catalogue of Variable stars (Samus et al. 2004). The periods for LP And, AFGL 3068, and IK Tau are from Men'shchikov et al. (2006), Le Bertre (1992), and González Delgado et al. (2003), respectively. Distances are derived by fitting the spectral energy distribution (SED) calculated with DUSTY (Sect. 6.1) to observed fluxes from the near-IR to the mm-range (Sect. 3.2 and Appendix B).

\section{Observational data}

\subsection{New observations of $\mathrm{CO}$ radio line emission}

When estimating the physical properties of the CSEs, e.g., massloss rates and kinetic temperatures, it is very important to have well-calibrated data for all lines (Schöier \& Olofsson 2001). CO 
Table 2. Main-beam efficiencies $\left(\eta_{\mathrm{mb}}\right)$, and main-beam FWHM: s $\left(\theta_{\mathrm{mb}}\right)$, at the frequencies of the $\mathrm{CO}$ lines.

\begin{tabular}{lcccc}
\hline \hline Transition & $\begin{array}{c}\text { Frequency } \\
{[\mathrm{GHz}]}\end{array}$ & $\eta_{\mathrm{mb}}$ & $\begin{array}{c}\theta_{\mathrm{mb}} \\
{\left[{ }^{\prime \prime}\right]}\end{array}$ & Telescope \\
\hline$J=1 \rightarrow 0$ & 115.271 & 0.43 & 33 & OSO \\
$J=2 \rightarrow 1$ & 230.538 & 0.66 & 21 & JCMT \\
$J=3 \rightarrow 2$ & 345.796 & 0.62 & 14 & JCMT \\
$J=4 \rightarrow 3$ & 461.041 & 0.5 & 11 & JCMT \\
$J=6 \rightarrow 5$ & 691.473 & 0.3 & 8 & JCMT \\
\hline
\end{tabular}

$J=1 \rightarrow 0(115.271 \mathrm{GHz})$ data was obtained in December 2003 using the Onsala Space Observatory (OSO) $20 \mathrm{~m}_{\text {telescope }}{ }^{1}$. The James Clerk Maxwell Telescope ${ }^{2}$ (JCMT) located on Mauna Kea was used to collect CO $J=2 \rightarrow 1(230.538 \mathrm{GHz})$, $J=3 \rightarrow 2(345.796 \mathrm{GHz}), J=4 \rightarrow 3(461.041 \mathrm{GHz})$, and $J=6 \rightarrow 5(691.473 \mathrm{GHz})$ line emission from the sample sources. The observations were performed in June, July, and October 2003.

All observations were made in a dual-beamswitch mode, where the source is alternately placed in the signal and the reference beam, using a beam throw of about $2^{\prime}$ at JCMT and $11^{\prime}$ at OSO. This method produces very flat baselines. Regular pointing checks made on strong continuum sources (JCMT) or SiO masers (OSO) were found to be consistent with the pointing model within $\approx 3^{\prime \prime}$.

The data was reduced in a standard way using $\mathrm{XS}^{3}$ by removing a first order polynomial fitted to the emission-free channel ranges and then binned in order to improve the signal-to-noise ratio. The intensity scale is given in main-beam-brightness temperature, $T_{\mathrm{mb}}=T_{\mathrm{A}}^{\star} / \eta_{\mathrm{mb}}$, where $T_{\mathrm{A}}^{\star}$ is the antenna temperature corrected for atmospheric attenuation using the chopper-wheel method, and $\eta_{\mathrm{mb}}$ is the main-beam efficiency. The adopted mainbeam efficiencies are given in Table 2 . The uncertainty in the absolute intensity scale is estimated to be about $\pm 20 \%$ for all lines except the $\mathrm{CO} J=6 \rightarrow 5$ lines, where we estimated a higher uncertainty of about $\pm 30 \%$.

The observed spectra are presented in Figs. 3, 4, and C.1-C.8, and velocity-integrated intensities are reported in Table 3.

\subsection{Dust continuum emission}

The SEDs are constructed from JHKLM-band, sub-millimetre, and millimetre photometric flux densities from the literature, together with IRAS data. SCUBA data (at 450 and $850 \mu \mathrm{m}$ ) was downloaded from the JCMT archive for CW Leo, AFGL 3068, and RW LMi. All fluxes and references are presented in Appendix B.

To check the dust radiative transfer model results, IRAS and ISO spectroscopic data were used. IRAS-LRS spectra were

\footnotetext{
1 The Onsala $20 \mathrm{~m}$ telescope is operated by the Swedish National Facility for Radio Astronomy, Onsala Space observatory at Chalmers University of technology.

2 The JCMT is operated by the Joint Astronomy Centre in Hilo, Hawaii on behalf of the present organizations: the Particle Physics and Astronomy Research Council in the United Kingdom, the National Research Council of Canada and the Netherlands Organization for Scientific Research.

${ }^{3} \mathrm{XS}$ is a package developed by P. Bergman to reduce and analyze a large number of single-dish spectra. It is publicly available from ftp://yggdrasil.oso. chalmers.se
}

Table 3. Integrated $\mathrm{CO}(J \rightarrow J-1)$ intensities, $\int T_{\mathrm{mb}} \mathrm{d} v$, in $\mathrm{K} \mathrm{km} \mathrm{s}^{-1}$.

\begin{tabular}{lrrrrrr}
\hline \hline \multirow{2}{*}{ Source } & OSO & \multicolumn{5}{c}{ JCMT } \\
\cline { 2 - 6 } \cline { 5 - 7 } & $1 \rightarrow 0$ & & $2 \rightarrow 1$ & $3 \rightarrow 2$ & $4 \rightarrow 3$ & $6 \rightarrow 5$ \\
\hline LP And & 63 & & 104 & $124^{a}$ & $143^{a}$ & 139 \\
CW Leo & 386 & 689 & $1070^{a}$ & $1230^{a}$ & $\ldots$ \\
RW LMi & 108 & & 123 & $244^{a}$ & $246^{a}$ & $\ldots$ \\
V384 Per & 35 & 63 & 40 & 79 & $\ldots$ \\
AFGL 3068 & 48 & 72 & 73 & 93 & 55 \\
TX Cam & 20 & 61 & 71 & 149 & $\ldots$ \\
GX Mon & 31 & 61 & 80 & 79 & $\ldots$ \\
WX Psc & 44 & 81 & 70 & 84 & 23 \\
IK Tau & 46 & 95 & $125^{a}$ & 130 & $\ldots$ \\
IRC-10529 & 16 & 45 & 27 & 22 & $\ldots$ \\
\hline
\end{tabular}

${ }^{a}$ JCMT archival data.

downloaded $^{4}$ for all stars in the sample. An absolute calibration correction was applied according to Volk \& Cohen (1989) and Cohen et al. (1992). The ISO LWS and SWS Highly Processed Data Products (HPDP) were downloaded and not processed further. LWS spectra were available for all stars except GX Mon. SWS spectra were found for TX Cam and WX Psc (SWS spectra for carbon stars were not searched for). For IK Tau, only a grating scan covering the wavelength range $\sim 29-49 \mu \mathrm{m}$ was available, and for IRC-10529, the SWS spectrum is very noisy above $28 \mu \mathrm{m}$.

\section{The circumstellar model}

The CSE is assumed to be spherically symmetric and formed by a constant mass-loss rate. It is assumed to be expanding at a constant velocity (derived from fitting the CO line widths) and to have a micro-turbulent velocity distribution with a doppler width of $0.5 \mathrm{~km} \mathrm{~s}^{-1}$ (e-folding value) throughout. In addition, a thermal contribution to the local line width is added, based on the derived kinetic temperature of the gas. The density structure is obtained from the conservation of mass.

Even in this simple model there are a number of uncertainties that will affect the strengths and the shapes of the CO lines and the shape of the SED, e.g., the thermodynamics of the gas, the CO chemistry, the dust composition, the optical properties of the dust, and the grain size distribution. In addition, there can be major deviations from this model due to e.g. non-isotropic, timevariable mass loss and a clumpy medium. Finally, the objects are long-period variables and the data are sometimes taken at different epochs. In many cases it may be impossible to disentangle which of these assumptions and effects, if any, are affecting the emerging radiation. This taken into account, the most reasonable approach is to choose the simplified circumstellar model and analyze all stars using the same method.

\section{CO line modelling}

Only a short description of the radiative transfer analysis of the circumstellar CO radio line emission is given here, since the adopted model has been described in detail in previous articles (Schöier \& Olofsson 2001, for carbon stars; and Olofsson et al. 2002, for M-type stars).

The non-LTE radiative transfer code is based on the Monte Carlo method. 41 rotational levels are included in each of the two

${ }^{4}$ http://www.iras.ucalgary.ca/ volk/getlrs_plot.html 
lowest vibrational states $(v=0$ and $v=1)$ in the excitation analysis of the $\mathrm{CO}$ molecule. Energy levels and radiative transition probabilities, as well as collisional rate coefficients for collisions between $\mathrm{CO}$ and $\mathrm{H}_{2}$, are taken from Schöier et al. (2005b) ${ }^{5}$. An ortho-to-para ratio of 3 is adopted when weighting together collisional rate coefficients for $\mathrm{CO}$ in collisions with ortho- and para$\mathrm{H}_{2}$. Radiation from the central source (assumed to be a blackbody), thermal dust grains (Sect. 6.1), and the cosmic microwave background is included.

The energy balance equation for the gas is solved selfconsistently, by including line cooling from $\mathrm{CO}$ and $\mathrm{H}_{2}$ and cooling due to the adiabatic expansion of the gas. The dominant gasheating mechanism is collisions between the $\mathrm{H}_{2}$ molecules and the dust grains. Photoelectric heating is also included and it is mostly important in the outer parts of the CSE. The free parameters describing the dust are combined in the parameter, $h$, defined as

$h=\left(\frac{\Psi}{0.01}\right)\left(\frac{2.0 \mathrm{~g} \mathrm{~cm}^{-3}}{\rho_{\mathrm{g}}}\right)\left(\frac{0.05 \mu \mathrm{m}}{a_{\mathrm{g}}}\right)$,

where $\Psi$ is the dust-to-gas mass-loss-rate ratio and $\rho_{\mathrm{g}}$ and $a_{\mathrm{g}}$ are the average density and radius of an individual dust grain, respectively. Following Schöier \& Olofsson (2001), we have adopted an average efficiency factor for momentum transfer (see Sect. 6.2), which is constant throughout the CSE, $\left\langle Q_{\mathrm{rp}}\right\rangle^{\dagger}=$ $3 \times 10^{-2}$. However, the particular choice of $\left\langle Q_{\mathrm{rp}}\right\rangle^{\dagger}$ is not so critical. As long as the product $h\left\langle Q_{\mathrm{rp}}\right\rangle^{\dagger 3 / 2}$ is kept constant (and $h$ is a free parameter), the model results are essentially the same for intermediate and high mass-loss-rate objects, for a given $\dot{M}$ (see Eqs. (4) and (6) in Schöier \& Olofsson 2001).

The radial distribution of $\mathrm{CO}$ is estimated using the model presented in Mamon et al. (1988). It includes photodissociation, shielding, and chemical exchange reactions. The photospheric abundance of $\mathrm{CO}$ relative to $\mathrm{H}_{2}$ is assumed to be $1 \times 10^{-3}$ for the carbon stars (Zuckerman \& Dyck 1986) and $2 \times 10^{-4}$ for the M-type stars (Kahane \& Jura 1994). The luminosity was estimated using a period-luminosity relation (see Sect. 2), and the inner radius of the CSE was taken from the SED fitting. It was shown in Schöier \& Olofsson (2001) that, in the intermediateto high-mass-loss-rate stars, the $\mathrm{CO}$ radio line intensities are essentially unaffected by a change in these parameters. The main reason is that in the higher-mass-loss-rate CSEs, CO is collisionally excited out to large radii. The mass-loss rate and $h$ are the remaining free parameters in the $\mathrm{CO}$ line modelling.

\section{Dust radiative transfer and dynamical model}

\subsection{Dust emission modelling}

Dust radiative transfer through a spherically symmetric envelope possesses scaling properties (Ivezic \& Elitzur 1997). This fact is put to use in the publicly available radiative transfer code DUSTY ${ }^{6}$ adopted here to model the observed continuum emission. The scaling properties of the solution makes DUSTY ideal for studying a large sample of stars, since only one model grid, although large, needs to be calculated for each dust type.

The most important parameter in the dust radiative transfer modelling is the dust optical depth

$\tau_{\lambda}=\kappa_{\lambda} \int_{r_{\mathrm{i}}}^{r_{\mathrm{e}}} \rho_{\mathrm{d}}(r) \mathrm{d} r$

\footnotetext{
5 http://www.strw. leidenuniv.nl/ moldata

6 http://www . pa . uky . edu/ moshe/dusty/
}

where $\kappa_{\lambda}$ is the dust opacity per unit mass, $\rho_{\mathrm{d}}$ is the dust mass density, and the integration is made from the inner $\left(r_{\mathrm{i}}\right)$ to the outer $\left(r_{\mathrm{e}}\right)$ radius of the CSE. Prompt dust formation is assumed at the inner radius. Amorphous carbon dust grains, with the optical constants presented in Suh (2000), are adopted for the carbon stars, and amorphous silicate grains are adopted for the M-type stars (Justtanont \& Tielens 1992). For simplicity, the dust grains are assumed to be of the same size with a radius of $0.1 \mu \mathrm{m}$ and have a density of $2 \mathrm{~g} \mathrm{~cm}^{-3}$ (carbon grains) or $3 \mathrm{~g} \mathrm{~cm}^{-3}$ (silicate grains).

In the modelling, the dust optical depth, specified at $10 \mu \mathrm{m}$, is varied in the range $0.1-10$ in steps of $\sim 10 \%$. In addition, the dust temperature at the inner radius of the dust envelope $T_{\mathrm{d}}\left(r_{\mathrm{i}}\right)$, and the stellar effective temperature $T_{\text {eff }}$, are varied. These are the three adjustable parameters in the analysis. The SED model result depends only weakly on the other input parameters, which are fixed at reasonable values. The size of the envelope is fixed at $r_{\mathrm{e}} / r_{\mathrm{i}}=20000$ to make sure that the whole envelope extent determined from the $\mathrm{CO}$ emission $\left(r_{\mathrm{p}}\right.$; see Table 3 in Schöier \& Olofsson 2001) is covered. Using an even larger size does not affect the result.

The temperature at the inner radius should be close to (or lower than) the dust condensation temperature. In the modelling it is varied in the range $300-1500 \mathrm{~K}$, in steps of $100 \mathrm{~K}$. The canonical dust condensation temperature of carbonaceous dust grains is $\approx 1500 \mathrm{~K}$, while the condensation temperature for the silicate grains is expected to be somewhat lower. However, prompt dust formation is an oversimplification. Given the complex chemistry close to the star, where shocks are present and grain growth is important, the dust-to-gas ratio probably depends on the radius. The dust temperature at the inner radius should therefore be treated as a measure of the characteristic radius where dust formation is essentially completed. The stellar effective temperature is varied in the range 1800-2400 K (also in steps of $100 \mathrm{~K}$ ), characteristic of high-mass-loss-rate AGB stars, but lower than that of AGB stars with optically thin dust envelopes (e.g., Lambert et al. 1986; Bergeat et al. 2001; Heras \& Hony 2005).

When scaling the result from DUSTY, the interstellar extinction is taken into account. It is calculated from the galactic longitude for each star, using Eq. (8) in Groenewegen et al. (1992).

\subsection{Dynamical model}

When light is scattered by a dust particle, some of its momentum is given to the scattering particle. For particles with an arbitrary shape, the efficiency factor for this momentum transfer depends on the orientation of the particle and on the polarization of the light. However, for spherical particles, as assumed here, it is independent of both. The dimensionless efficiency factor is given by the momentum fraction taken from the incident beam divided by the geometrical cross section of a particle:

$$
Q_{\lambda}=\frac{4}{3} \kappa_{\lambda} a_{\mathrm{g}} \rho_{\mathrm{g}}
$$

In the CSEs of AGB stars, momentum transfer from photons to dust grains pushes the dust outward, and friction between the dust and gas particles determines the gas outflow velocity. By assuming that the wind is entirely dust driven, conservation of 
mass and momentum can be used to set up equations for the velocity of the dust $\left(v_{\mathrm{d}}\right)$ and the gas $\left(v_{\mathrm{g}}\right)$ particles,

$\frac{\mathrm{d} v_{\mathrm{d}}^{2}}{\mathrm{~d} r}=\frac{2}{r^{2}}\left[\frac{\sigma_{\mathrm{d}}\left\langle Q_{\mathrm{rp}}\right\rangle L_{*}}{4 \pi c m_{\mathrm{d}}}-G M_{*}-\frac{\sigma_{\mathrm{d}} \dot{M} v_{\mathrm{dr}} \sqrt{\left(v_{\mathrm{s}}^{2}+v_{\mathrm{rel}}^{2}\right)}}{4 \pi m_{\mathrm{d}} v}\right]$

$\frac{\mathrm{d} v_{\mathrm{g}}^{2}}{\mathrm{~d} r}=\frac{2}{r^{2}}\left[-G M_{*}+\frac{\Psi \sigma_{\mathrm{d}} \dot{M} v_{\mathrm{dr}} \sqrt{\left(v_{\mathrm{s}}^{2}+v_{\mathrm{rel}}^{2}\right)}}{4 \pi m_{\mathrm{d}} v_{\mathrm{d}}}\right]$,

where $\sigma_{\mathrm{d}}$ and $m_{\mathrm{d}}$ are the collisional cross section and mass of a dust grain, $\left\langle Q_{\mathrm{rp}}\right\rangle$ is the radiation pressure efficiency averaged over the photon spectrum, $L_{*}$ and $M_{*}$ are the stellar luminosity and mass, $v_{\mathrm{dr}}\left(=v_{\mathrm{d}}-v_{\mathrm{g}}\right)$ is the drift velocity between the dust and the gas, and $v_{\mathrm{s}}$ is the sound speed assumed to be $2 \mathrm{~km} \mathrm{~s}^{-1}$ (Lamers \& Cassinelli 1999). A stellar mass of $1.5 M_{\odot}$ is used for all stars in the sample (Kahane et al. 2000; Olivier et al. 2001).

The radiation pressure efficiency $Q_{\lambda}$ is determined and averaged over the photon spectrum,

$\left\langle Q_{\mathrm{rp}}\right\rangle(r)=\frac{\int Q_{\lambda} B_{\lambda} \mathrm{e}^{-\tau_{\lambda}} \mathrm{d} \lambda}{\int B_{\lambda} \mathrm{e}^{-\tau_{\lambda}} \mathrm{d} \lambda}$,

where $B_{\lambda}$, calculated from the effective temperature of the star, is weighted by the absorption determined by the optical depth $\tau_{\lambda}$ derived using the dust opacity and the dust density structure from the dust emission modelling (Eq. (2)). This includes absorption, but not re-emission at longer wavelengths. This simplification will probably not have a big effect on the result due to the wavelength dependence on the radiation pressure efficiency. DUSTY includes an option where the wind structure can be calculated by solving the hydrodynamical equations. The drawback compared to the dynamical model presented here is that a dust-to-gas ratio needs to be assumed in order to get an estimate of the mass-loss rate, but as opposed to our model, it includes the re-emission by the dust. A comparison between the results from our dynamical model and the mass-loss-rate estimates derived by DUSTY on five of our sources showed that both methods give the same result (within $\sim 30 \%$ ) for the same dust-to-gas ratio.

$\left\langle Q_{\mathrm{rp}}\right\rangle$ rapidly decreases and reaches its terminal value, $\left\langle Q_{\mathrm{rp}}\right\rangle^{\dagger}$, at $\approx 20-30 R_{\star}$. Equations (4) to (6) are solved in an iterative way, for different mass-loss rates, until the dust density structure is consistent with the optical depth derived in the dust radiative transfer model, and a fit to the observed gas expansion velocity is found. In this way, an independent estimate of the mass-loss rate is obtained.

Bains et al. (2003) investigated maser emission towards four AGB stars and derived velocity profiles for their circumstellar envelopes. In Fig. 1, our result for IK Tau is overlayed and shown to be consistent with their data.

\section{Results}

\subsection{CO line modelling}

\subsubsection{Strategy for finding the best-fit model}

Only two parameters are adjustable when fitting the model to the observed integrated line intensities: the mass-loss rate, $\dot{M}$, and the $h$-parameter. Models are calculated for a large number of mass-loss rates and $h$-parameters for each star and the best-fit

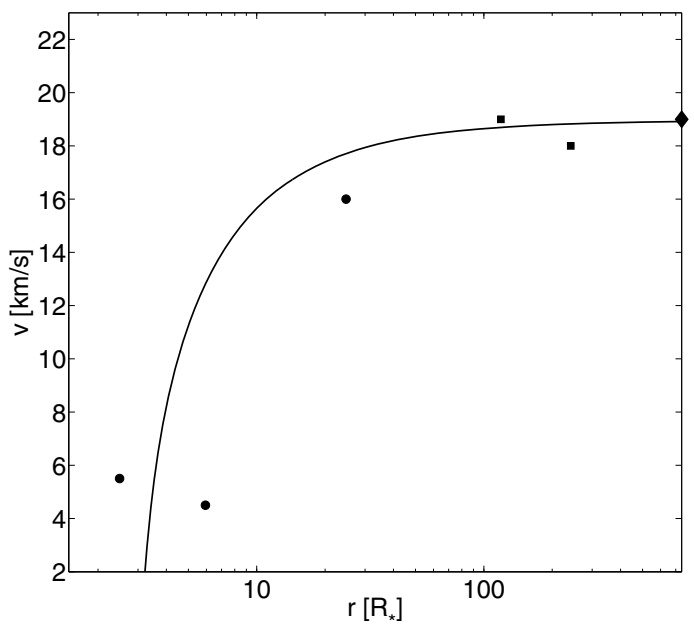

Fig. 1. The velocity profile for IK Tau from the dynamical model overlayed with the velocity data obtained from mapping of maser emission (see Bains et al. 2003, and references therein). $\mathrm{H}_{2} \mathrm{O}$ masers (circles) and $\mathrm{OH}$ maser (squares) have been used. The expansion velocity derived from the CO data is marked by a diamond.

model is found using $\chi^{2}$-statistics. The reduced $\chi^{2}$ for the best-fit model is estimated from

$\chi_{\text {red }}^{2}=\frac{\chi_{\min }^{2}}{N-p}$,

where $N$ is the number of observational constraints, and $p$ is the number of adjustable parameters ( 2 in our case). $\chi_{\min }^{2}$ is the minimum $\chi^{2}$ obtained in the model runs, which is calculated as,

$\chi^{2}=\sum_{i=1}^{N} \frac{\left(I_{\mathrm{mod}, i}-I_{\mathrm{obs}, i}\right)^{2}}{\sigma_{i}^{2}}$,

where $\sigma$, for the high-quality data presented here, is dominated by the calibration uncertainties assumed to be $20 \%$ for all lines except the $J=6 \rightarrow 5$ lines where they are assumed to be $30 \%$ (see Sect. 3.1).

\subsubsection{Mass-loss rates from $\mathrm{CO}$}

We are able to successfully model the CO line emission from all stars in our sample, both carbon stars and M-type stars, using a constant mass-loss rate. (The $J=6 \rightarrow 5$ line of WX Psc poses a problem that will be further discussed in Sect. 8.2.3.) The results are presented in Table 4 . Figure 2 shows $\chi^{2}$-maps for all our sample stars. The difficulty in setting an upper limit on the mass-loss rate for high-mass-loss-rate stars using $\mathrm{CO}$ radio line intensities only is apparent. The saturation of the optically thick CO lines weakens significantly the dependence of the line intensity on the amount of $\mathrm{CO}$ in the envelope.

In some cases, better constraints on the mass-loss rate can be set by including the shape of the line profile. Changing the massloss rate results in a changing photodissociation radius (see, e.g., model by Stanek et al. 1995), and this may result in a change of the line profile. Visual inspection of the line profiles (within the range of the innermost contour of the $\chi^{2}$-maps) was used to pick the best model presented in Table 4 and when determining the error bars in Fig. 7. In principle, it is possible to account for the shape of the line profile in a more quantitative way, i.e. by dividing the line into several velocity bins and calculating an error using $\chi^{2}$-statistics. However, this is problematic since the resulting $\chi^{2}$-values become very sensitive to very small variations in 
Table 4. Mass-loss rates, $h$-parameters, and terminal gas expansion velocities $v_{\mathrm{e}}$, derived by fitting the $\mathrm{CO}$ line radiative transfer model to the observations.

\begin{tabular}{lccccc}
\hline \hline Source & $\begin{array}{c}\dot{M} \\
{\left[10^{-6} M_{\odot} \mathrm{yr}^{-1}\right]}\end{array}$ & $\begin{array}{c}h \\
{\left[\mathrm{~km} \mathrm{~s}^{-1}\right]}\end{array}$ & $v_{\mathrm{e}}$ & $\chi_{\text {red }}^{2}$ & $N$ \\
\hline LP And & 7 & 2.0 & 14.0 & 0.5 & 5 \\
CW Leo & 20 & 1.0 & 14.5 & 0.1 & 4 \\
RW LMi & 5 & 1.5 & 17.0 & 0.7 & 4 \\
V384 Per & 3 & 1.0 & 14.5 & 2.7 & 4 \\
AFGL 3068 & 20 & 2.0 & 14.0 & 0.9 & 5 \\
& & & & & \\
TX Cam & 7 & 1.0 & 18.5 & 3.0 & 4 \\
GX Mon & 20 & 0.5 & 18.7 & 0.1 & 4 \\
WX Psc & 40 & 0.4 & 19.3 & 1.0 & 4 \\
IK Tau & 10 & 0.3 & 19.0 & 0.4 & 4 \\
IRC-10529 & 16 & 0.2 & 14.0 & 3.8 & 4 \\
\hline
\end{tabular}

the line-profile shape which may not be possible to account for by changing the free parameters in the adopted model. Also, in cases like TX Cam (Fig. 4), a fit to the line-profile shape can never be found within the adopted model, either the adopted distance or the photodissociation radius must be changed (see Sect. 8.2.1 for a discussion of this).

Figures 3 and 4 give the comparisons between the observed and the best-fit model CO lines for the carbon star LP And and the M-type star TX Cam, respectively. These results are discussed in Sects. 8.1.1 and 8.2.1, respectively. The results for the remaining stars are shown in Appendix C. The differences between the integrated line intensities in the best-fit model and the observed lines are presented in Table C.1.

\subsection{Dust emission modelling}

\subsubsection{Strategy for finding the best-fit model}

The observational constraints in the form of SEDs, covering the wavelength range $\sim 1-1300 \mu \mathrm{m}$, are analyzed using $\chi^{2}$-statistics. When combining the measured fluxes into an SED, the variability of the star must be taken into account. This is particularly important for fluxes measured at shorter wavelengths, where the amplitude of the variation is larger. To assess how strongly the fluxes are affected, the mean and deviation was calculated from several measurements performed at different epochs. As a result a $60 \%$ uncertainty was assumed for the $J$-band $(1.24 \mu \mathrm{m}), 50 \%$ for the $H$-band $(1.63 \mu \mathrm{m}), 40 \%$ for the $K$-band $(2.19 \mu \mathrm{m})$, and $30 \%$ for the $L$ - and $M$-bands (3.79 and $4.64 \mu \mathrm{m}$, respectively). At wavelengths longer than $5 \mu \mathrm{m}$, a calibration uncertainty of $20 \%$ was added to the total error budget and it dominates the error (since there is limited information on the variability at these wavelengths). In some cases where there are many fluxes measured at roughly the same wavelength, we introduce an average value to avoid a bias in the $\chi^{2}$ calculation at certain wavelengths. (This is the reason why some of the fluxes presented in Table B.1 are not included in the analysis.) The SEDs constructed from the photometric flux densities are thus averages, where the variability of the star at different wavelengths is reflected in the error bars (typically larger uncertainties at shorter wavelengths). The best-fit model is found from minimizing

$\chi^{2}=\sum_{i=1}^{N}\left[\frac{\left(F_{\mathrm{mod}, \lambda}-F_{\mathrm{obs}, \lambda}\right)}{\sigma_{\lambda}}\right]^{2}$,
Table 5. Results from the dust emission modelling.

\begin{tabular}{lccccc}
\hline \hline Source & $\begin{array}{c}T_{\star} \\
{[\mathrm{K}]}\end{array}$ & $\begin{array}{c}T_{\mathrm{d}}\left(r_{\mathrm{i}}\right) \\
{[\mathrm{K}]}\end{array}$ & $\tau_{10}$ & $\chi_{\text {red }}^{2}$ & $N$ \\
\hline LP And & 2400 & 1100 & 0.55 & 1.6 & 11 \\
CW Leo & 2000 & 1500 & 1.2 & 2.4 & 12 \\
RW LMi & 2400 & 1200 & 0.6 & 0.9 & 11 \\
V384 Per & 1800 & 1000 & 0.2 & 0.9 & 10 \\
AFGL 3068 & 2400 & 1500 & 4.0 & 5.3 & 11 \\
& & & & & \\
TX Cam & 2200 & 800 & 0.4 & 0.5 & 9 \\
GX Mon & 1800 & 500 & 0.3 & 4.9 & 9 \\
WX Psc & 1800 & 1000 & 3.0 & 1.1 & 12 \\
IK Tau & 2400 & 1200 & 1.2 & 0.9 & 12 \\
IRC-10529 & 1800 & 800 & 3.0 & 3.1 & 10 \\
\hline
\end{tabular}

where $F_{\lambda}$ is the flux density and $\sigma_{\lambda}$ the uncertainty in the measured flux density at wavelength $\lambda$, and the summation is done over all $N$ independent observations.

\subsubsection{Results from dust emission modelling}

Table 5 lists the results from the dust modelling, the dust temperature at the inner radius $T_{\mathrm{d}}\left(r_{\mathrm{i}}\right)$, dust optical depth at $10 \mu \mathrm{m}$ $\tau_{10 \mu \mathrm{m}}, \chi_{\text {red }}^{2}$ from fitting the SED, and the number of observational constraints $N \cdot \chi^{2}$-maps, at constant best-fit stellar effective temperature, showing the level of consistency between the modelled SED and the photometric fluxes, when varying the dust temperature at the inner radius and the optical depth at $10 \mu \mathrm{m}$, are displayed in Fig. 5. The SEDs from the dustradiative-transfer model, with the observed photometric fluxes, are shown in Fig. 6. $\chi_{\text {red }}^{2}$ are generally close to 1 and the dust optical depth at $10 \mu \mathrm{m}$ is rather well constrained for most sources (see Fig. 5).

\subsubsection{IR spectra - a consistency check}

Low resolution spectra in the wavelength range 7.5-23 $\mu \mathrm{m}$ covering the silicate features at around 10 and $20 \mu \mathrm{m}$, were measured with the Low Resolution Spectrograph (LRS) as part of the IRAS mission. Later, the Short Wavelength Spectrometer (SWS) and the Long Wavelength Spectrometer (LWS) onboard ISO measured medium and high resolution spectra in the wavelength range $2.38-45.2 \mu \mathrm{m}$ and $43-197 \mu \mathrm{m}$, respectively. Short wavelength spectrometer spectra also cover the silicate features and LWS spectra show the slope of the SED at longer wavelengths and is particularly sensitive to the cold dust.

The strengths of the silicate features are very sensitive to the dust optical depth (see for instance Fig. 1 in van Loon 2006) and in turn the dust mass-loss rate. As a consistency check, the IRAS-LRS spectra and SWS spectra are plotted for the M-type stars in the lowest panel of Fig. 6. The carbon stars all have the SiC feature at $11 \mu \mathrm{m}$ (e.g., Speck et al. 1997). However, since SiC grains compose a small fraction of the total dust content $(\sim 10 \%)$ and have a minor effect on the overall SED except for the $11 \mu \mathrm{m}$ feature (Suh 2000), we have not included SiC in the dust model and consequently the observed spectra for the carbon stars are not shown. LWS spectra are available for all stars but one, and they are also plotted in Fig. 6.

A fit to the LRS and SWS spectra has not been attempted because of the difficulty in including them in a proper best-fit analysis. One complication is that the spectral shape and strength of the $9.7 \mu \mathrm{m}$ silicate feature can change with the pulsational 

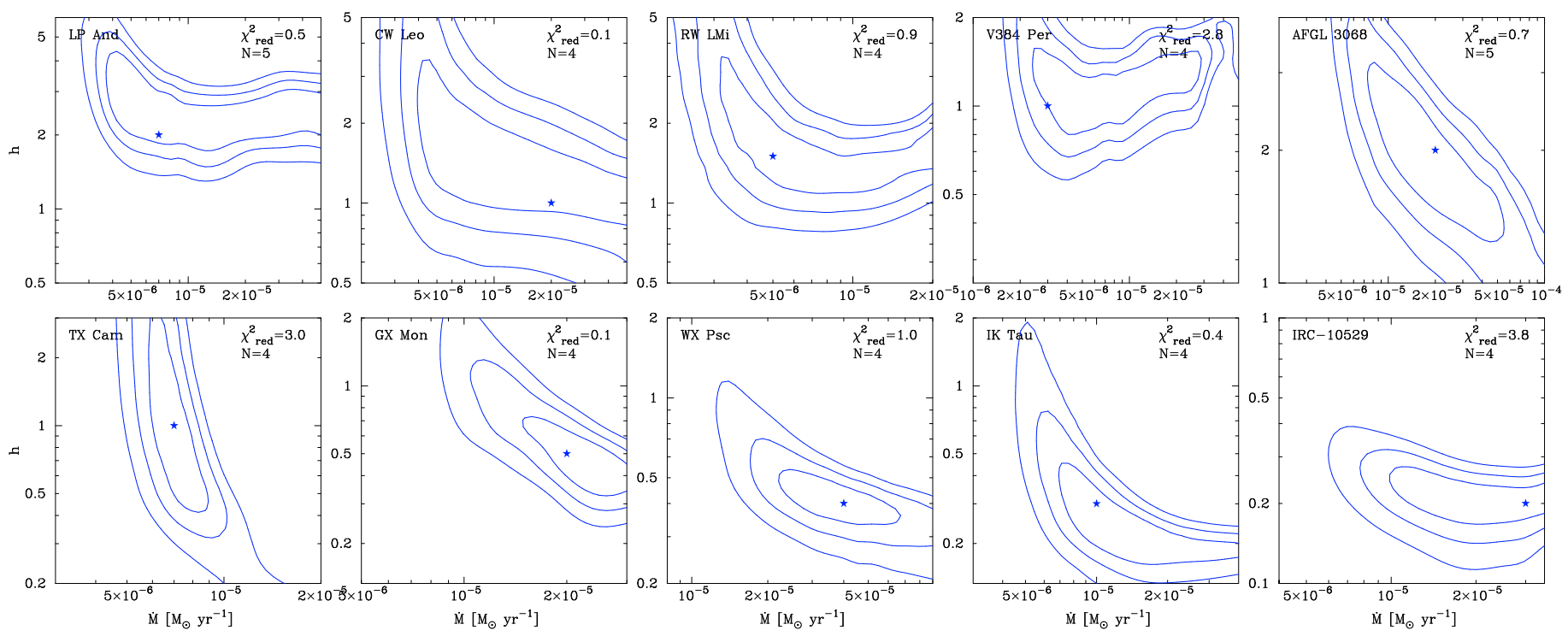

Fig. 2. $\chi^{2}$-maps showing the level of consistency between the velocity-integrated CO line intensity from the observations and the line model when varying the mass-loss rate and the $h$-parameter. The innermost contour corresponds to a $68 \%$ confidence level. The star indicates the best-fit model. The shapes of the line profiles were taken into account when choosing the best-fit model.
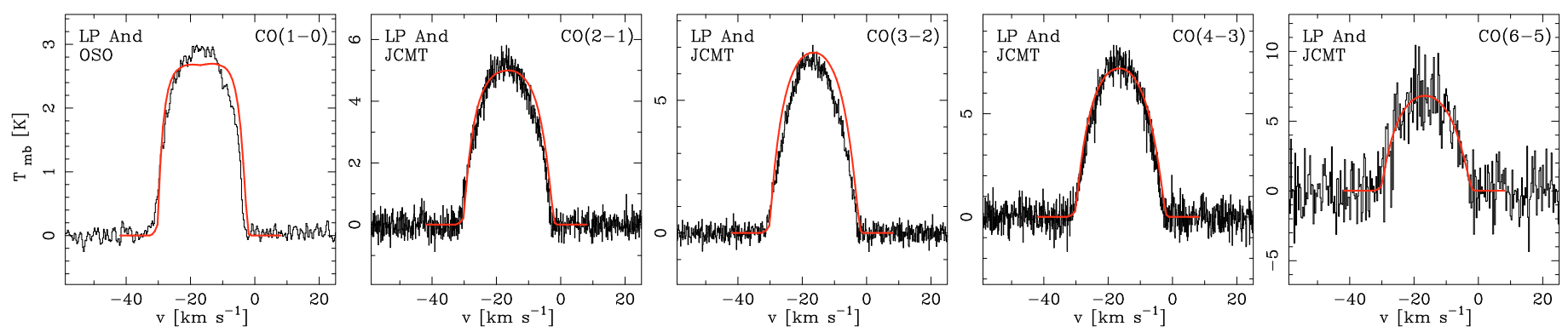

Fig. 3. The observed CO $J=1 \rightarrow 0$ line from OSO and the $2 \rightarrow 1,3 \rightarrow 2,4 \rightarrow 3$, and $6 \rightarrow 5$ lines from JCMT (histogram) of the carbon star LP And, overlayed with the best-fit model $\left(\dot{M}=7 \times 10^{-6} M_{\odot} \mathrm{yr}^{-1}\right)$ lines (solid line) from the radiative transfer modelling. The reduced $\chi^{2}$ for this model is 0.25. See Sect. 8.1.1.
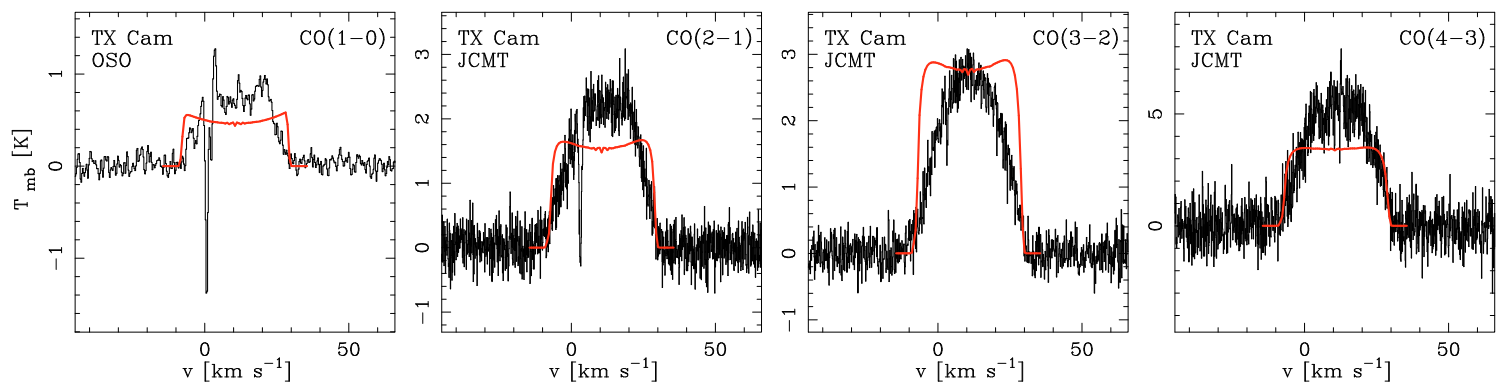

Fig. 4. The observed CO $J=1 \rightarrow 0$ line from OSO and the $2 \rightarrow 1,3 \rightarrow 2$, and $4 \rightarrow 3$ lines from JCMT (histogram) of the M-type star TX Cam, overlayed with the best-fit model $\left(\dot{M}=7 \times 10^{-6} M_{\odot} \mathrm{yr}^{-1}\right)$ lines (solid line) from the radiative transfer modelling. The reduced $\chi^{2}$ for this model is 3.0. See Sect. 8.2.1.

phase in stars with clear features and definite bolometric variability (Monnier et al. 1998). This is likely valid for all M-type stars in our sample. Also, since these spectra are reasonably well fitted (see Fig. 6), we expect the change in the resulting dust optical depth and inner radius, if the spectra were fitted in more detail (such as silicate features), to be small. A small change in the optical depth will result in an even smaller change in the estimated mass-loss rate from the dynamical model (see Table 7).

For some stars (CW Leo, RW LMi, AFGL 3068, and IRC-10529) there is a slight discrepancy between the slope of the LWS spectrum and that of the best-fit model. To perfectly reproduce the slope of the LWS spectra and the photometric fluxes at longer wavelengths, a varying mass-loss rate of these stars would probably have to be invoked. This has already been suggested for CW Leo by Groenewegen (1997), who found that the dust density far from the star was underestimated by the best-fit $r^{-2}$ model. Other possible causes could be a difference between the optical properties of the model dust and the actual dust surrounding the star (see e.g. Figs. 1 and 6 in Suh 2000), or the uncertainty in the long-wavelength dependence of the dust opacity. The fluxes beyond $\sim 800 \mu \mathrm{m}$ might also be affected by other processes than the dust emission, e.g., free-free emission 

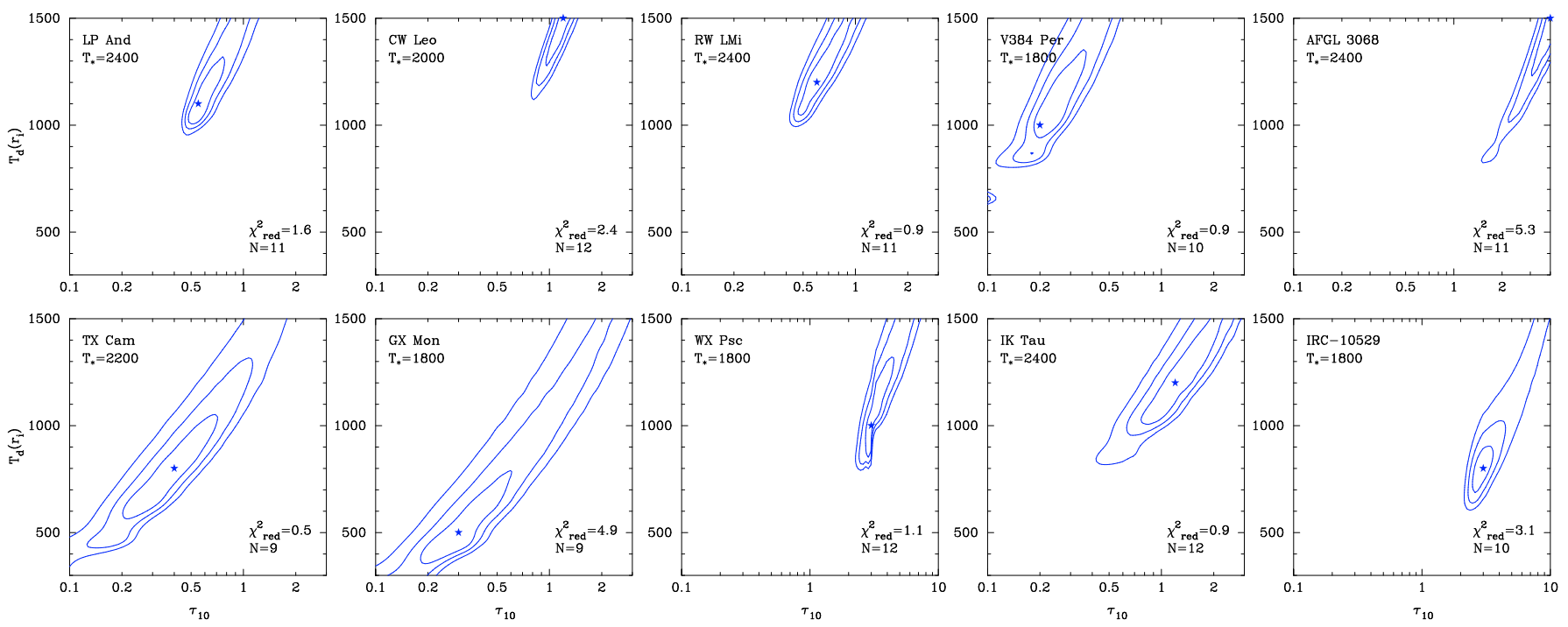

Fig. 5. $\chi^{2}$-maps showing the level of consistency between the photometric fluxes and the dust-continuum model when varying $\tau_{10} \mu \mathrm{m}$ and $T_{\mathrm{d}}$, at constant $T_{\text {eff. }}$. The innermost contour corresponds to a $68 \%$ confidence level. The star indicates the best-fit model chosen using the strategy described in Sect. 7.2.1. All grids are terminated at $T_{\mathrm{d}}\left(r_{\mathrm{i}}\right)=1500 \mathrm{~K}$ since a dust temperature at the inner radius larger than this is unlikely. This determines the best-fit models in the cases of CW Leo and AFGL 3068.
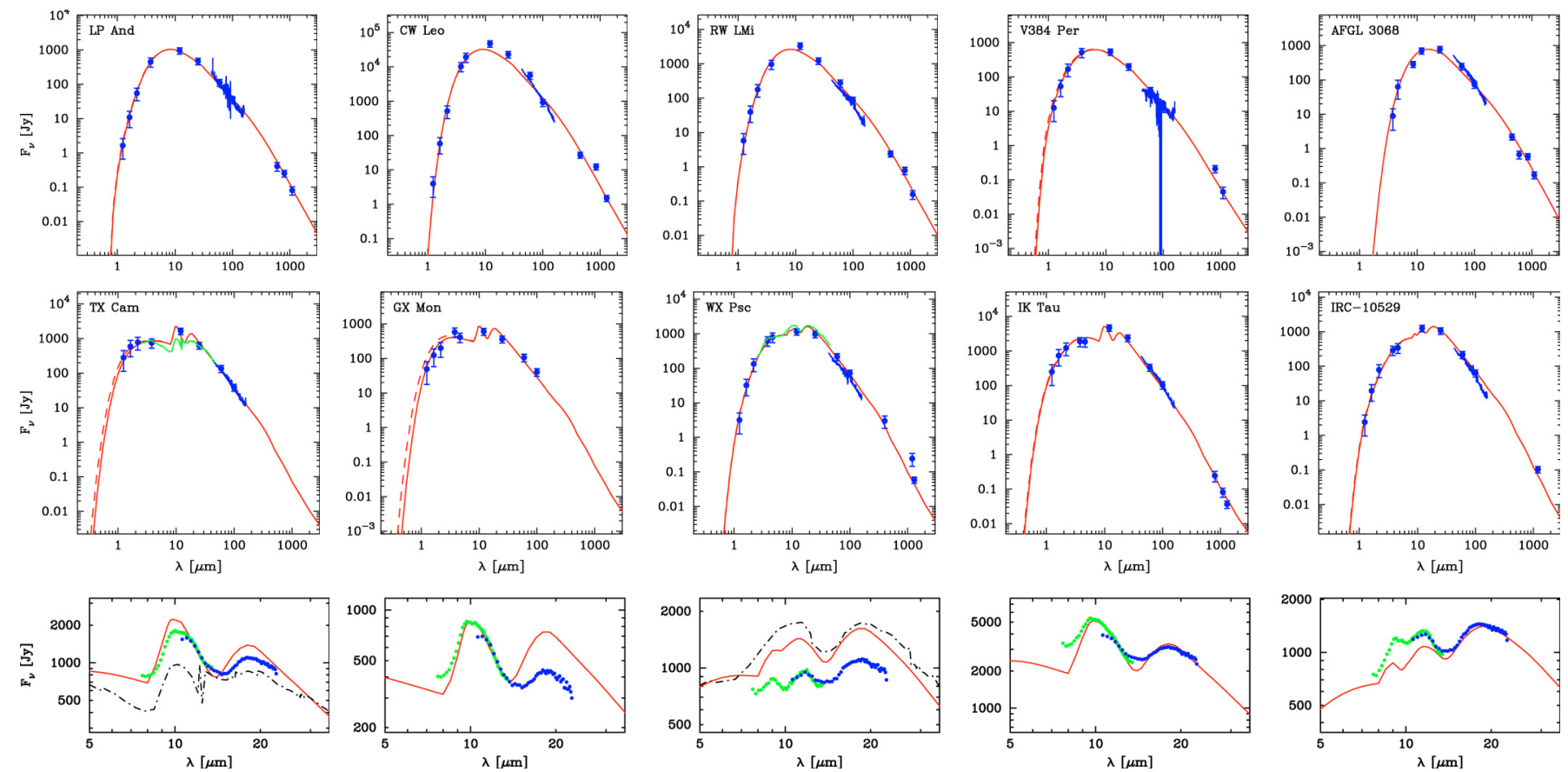

Fig. 6. Observed photometric fluxes together with ISO-LWS and ISO-SWS spectra overlayed by the best-fit model SEDs derived from the dust radiative transfer model. The model is represented by the solid line in all panels. The dashed line shows the model SED when the interstellar extinction is not taken into account. The ISO-LWS spectra are plotted for all stars but GX Mon. The lower panels show the wavelength interval 5-35 $\mu \mathrm{m}$ in more detail for the M-type stars. The best-fit models are here shown together with the observed IRAS-LRS spectra (dotted line) and the ISO-SWS spectra (dot-dashed line) when available.

and molecular line emission (Groenewegen 1997; Dehaes et al. 2007). Bearing in mind the uncertainties in the circumstellar model (Sect. 4) and our aim to analyze all stars with the same assumptions we find that the LRS, SWS, and LWS data are well reproduced by our best-fit models.

\subsubsection{Mass-loss-rates from dust continuum and dynamical model}

In the dynamical model, the density structure is calculated from the conservation of mass and the model is iterated until this gives an optical depth that agrees with the dust continuum optical depth obtained from the SED modelling (within one percent) and the resulting terminal gas expansion velocity from solving Eqs. (4) and (5) corresponds to the observed expansion velocity within $0.1 \%$.

Table 6 lists mass-loss rates, $v_{\mathrm{dr}}, \Psi$, and $\left\langle Q_{\mathrm{rp}}\right\rangle^{\dagger}$ for the best-fit dynamical model.

\section{Individual objects}

All sources in the sample are well-studied both at IR and radio wavelengths. As established already in Sects. 7.1 and 7.2, we are 
Table 6. Mass-loss rates and other quantities from the dynamical model.

\begin{tabular}{lccccc}
\hline \hline Source & $\dot{M}$ & $v_{\mathrm{dr}}$ & $\Psi$ & $\left\langle Q_{\mathrm{rp}}\right\rangle^{\dagger}$ & $\dot{M}_{\mathrm{dyn}} / \dot{M}_{\mathrm{CO}}$ \\
& {$\left[10^{-6} M_{\odot} \mathrm{yr}^{-1}\right]$} & {$\left[\mathrm{km} \mathrm{s}^{-1}\right]$} & {$\left[10^{-3}\right]$} & & \\
\hline LP And & 17 & 3.1 & 1 & 0.09 & 2.5 \\
CW Leo & 21 & 1.9 & 2 & 0.05 & 1.1 \\
RW LMi & 17 & 1.3 & 2 & 0.08 & 3.3 \\
V384 Per & 5 & 6.5 & 2 & 0.11 & 1.6 \\
AFGL 3068 & 62 & 0.7 & 3 & 0.02 & 3.1 \\
& & & & & \\
TX Cam & 3 & 10.6 & 4 & 0.11 & 0.5 \\
GX Mon & 2 & 12.6 & 10 & 0.09 & 0.1 \\
WX Psc & 18 & 4.3 & 3 & 0.06 & 0.5 \\
IK Tau & 7 & 7.3 & 2 & 0.10 & 0.7 \\
IRC-10529 & 26 & 2.9 & 2 & 0.06 & 1.6 \\
\hline
\end{tabular}

able to reasonably successfully model the CO line emission (the integrated intensities and the line shapes) and the SEDs for all 10 stars using a constant mass-loss rate.

In the following discussion it should be kept in mind that the different methods measure the mass-loss rate at different epochs and over different time intervals. The size of the CO line emitting region decreases with the $J$-number and hence the different lines measure the mass-loss rate at slightly different epochs. The same is true for the SEDs where the emission at different wavelenghts (apart from the photospheric contribution) comes from different parts of the CSE. The estimated mass-loss rate should be considered as an average and it is a complicated synthesis of the different epochs and time scales.

\subsection{Carbon stars}

\subsubsection{LP And}

The derived mass-loss rate from the $\mathrm{CO}$ modelling is $7 \times$ $10^{-6} M_{\odot} \mathrm{yr}^{-1}$, while the dynamical model gives a mass-loss rate of $1.7 \times 10^{-5} M_{\odot} \mathrm{yr}^{-1}$, a factor of 2.5 larger. The model lines agree very well with all five observed lines, $\chi_{\text {red }}^{2}=0.25$ (Fig. 3 ). Previous mass-loss-rate estimates from CO radio lines have resulted in mass-loss rates close to our results, $1.2 \times 10^{-5} M_{\odot} \mathrm{yr}^{-1}$ $\left(\mathrm{CO} / \mathrm{H}_{2}=1.1 \times 10^{-3}\right)$ (Teyssier et al. 2006), and $1.5 \times$ $10^{-5} M_{\odot} \mathrm{yr}^{-1}$ (Schöier \& Olofsson 2001), both assuming a distance of $630 \mathrm{pc}$. Men'shchikov et al. (2006) estimated the massloss rate to be $1.9 \times 10^{-5} M_{\odot} \mathrm{yr}^{-1}$ from a dust radiative transfer model, assuming a distance of $740 \mathrm{pc}$ and a dust-to-gas mass ratio of 0.0039 . The corresponding mass-loss rate at the distance used and the dust-to-gas ratio obtained in our model, would be $1.1 \times 10^{-5} M_{\odot} \mathrm{yr}^{-1}$, in fairly good agreement with our result.

Neri et al. (1998) found a slight asymmetry in the CSE from maps of the $\mathrm{CO}(J=1 \rightarrow 0)$ emission. Mauron \& Huggins (2006), using optical images in scattered light, suggested that the envelope around LP And may have an interesting structure, but concluded that the signal-to-noise in their data is too low to make any firm statement.

\subsubsection{CW Leo $(\mathrm{IRC}+10216)$}

Being the closest carbon star, CW Leo is certainly the most wellstudied. We estimate the mass-loss rate to be $2 \times 10^{-5} M_{\odot} \mathrm{yr}^{-1}$ from the $\mathrm{CO}$ line modelling and $2.1 \times 10^{-5} M_{\odot} \mathrm{yr}^{-1}$ from the dynamical model. Previous estimates of its mass-loss rate from $\mathrm{CO}$ is $1.2 \times 10^{-5} M_{\odot} \mathrm{yr}^{-1}$ (Teyssier et al. 2006) and $1.5 \times 10^{-5} M_{\odot} \mathrm{yr}^{-1}$ (Schöier \& Olofsson 2001), both assuming a distance of $120 \mathrm{pc}$. Groenewegen et al. (1998) did a very thorough investigation of the $\mathrm{CO}$ radio line emission toward $\mathrm{CW}$ Leo and estimated the present day mass-loss rate to $(1.5 \pm 0.3) \times$ $10^{-5} M_{\odot} \mathrm{yr}^{-1}$ and the distance to lie between 110 and $135 \mathrm{pc}$.

Several reports on structures in the circumstellar environment around CW Leo can be found in the literature (e.g., Mauron \& Huggins 1999; Fong et al. 2006). Optical images show arc-like structures on arcsecond scales in the CSE, but whether this is due to changes in the mass-loss rate over time, is still under debate (e.g., Leão et al. 2006, and references therein).

\subsubsection{V384 Per}

The best-fit $\mathrm{CO}$ model gives a mass-loss rate of $3 \times$ $10^{-6} M_{\odot} \mathrm{yr}^{-1}$. However, as can be seen in Fig. C.3, the model $J=1 \rightarrow 0,2 \rightarrow 1$ and $4 \rightarrow 3$ lines are all 15-20\% weaker than the observed lines, while the model $J=3 \rightarrow 2$ line is $65 \%$ stronger than the observed line. This results in a rather high $\chi_{\text {red }}^{2}=2.8$. The most likely explanation is a calibration error in the $J=3 \rightarrow 2$ line. The mass-loss rate from the dynamical model is higher, $\dot{M}=5 \times 10^{-6} M_{\odot} \mathrm{yr}^{-1}$. Schöier \& Olofsson (2001) estimated $\dot{M}=3.5 \times 10^{-6} M_{\odot} \mathrm{yr}^{-1}$ at $560 \mathrm{pc}$ from CO line modelling.

\subsubsection{AFGL 3068 (LL Peg)}

Our model of the CO line emission reproduces all five lines for a constant mass-loss rate of $2 \times 10^{-5} M_{\odot} \mathrm{yr}^{-1}$ with a $\chi_{\text {red }}^{2}=$ 0.7. The dynamical model gives $\dot{M}=6.2 \times 10^{-5} M_{\odot} \mathrm{yr}^{-1}$, slightly more than a factor of three higher. Earlier estimates of the mass-loss rate from $\mathrm{CO}$ line modelling have resulted in $\dot{M}=6.0 \times 10^{-5} M_{\odot} \mathrm{yr}^{-1}$ (Teyssier et al. 2006) at $1000 \mathrm{pc}$ and $2.0 \times 10^{-5} M_{\odot} \mathrm{yr}^{-1}$ (Woods et al. 2003) at $820 \mathrm{pc}$. Teyssier et al. (2006) deduced a much lower temperature in the outer envelope than we calculate in our model and this is the reason for the discrepancy between the estimates.

There is no doubt that the CSE around this source is asymmetric and have density structures that cannot be reconciled with a spherically symmetric CSE (Mauron \& Huggins 2006). Radial CO $(J=1 \rightarrow 0)$ visibility profiles show a compact inner envelope surrounded by a large outer shell. The outer shell is strongly bipolar and there are even indications that it might be detached (Neri et al. 1998).

\subsubsection{RW LMi (CIT 6)}

We find the CO line data to be consistent with a mass-loss rate of $5 \times 10^{-6} M_{\odot} \mathrm{yr}^{-1}$. From the dynamical model the mass-loss rate is estimated to be $17 \times 10^{-6} M_{\odot} \mathrm{yr}^{-1}$. Teyssier et al. (2006) found it necessary to infer a change in the mass-loss rate about 250 years ago to fit the $\mathrm{CO}$ intensity measured at offset positions; the mass-loss rate has decreased from $7.5 \times 10^{-6} M_{\odot} \mathrm{yr}^{-1}$ to $5.0 \times 10^{-6} M_{\odot} \mathrm{yr}^{-1}$. The two component structure was already noticed by Neri et al. (1998) when first inspecting the interferometric data. Schöier \& Olofsson (2001) estimated $\dot{M}=$ $6.0 \times 10^{-6} M_{\odot} \mathrm{yr}^{-1}$ from CO line modelling using the same distance as Teyssier et al. (2006), 440 pc. 


\subsection{M-type stars}

\subsubsection{TX Cam}

The best-fit $\mathrm{CO}$ model gives $\dot{M}=7 \times 10^{-6} M_{\odot} \mathrm{yr}^{-1}$ (the same rate was derived by González Delgado et al. 2003), but the model does not reproduce the shape of the observed line profiles (Fig. 4). The observed $J=1 \rightarrow 0$ and $2 \rightarrow 1$ lines are flat-topped, which suggests unresolved, optically thin emission, while the model gives double-peaked lines, which suggests resolved, optically thin emission. The observed $J=3 \rightarrow 2$ and $4 \rightarrow 3$ lines are parabolic and thus optically thick, while the model produces an optically thin, resolved $J=3 \rightarrow 2$ line and a flat-topped $4 \rightarrow 3$ line. The simplest explanation would be that the distance is underestimated. A larger distance requires a higher mass-loss rate to reproduce the line intensities and hence gives line profiles that better reproduce the observed ones. An increased distance also decreases the angular size, but this is partly counteracted by the fact that the $\mathrm{CO}$ photodissociation radius increases with the mass-loss rate. Another explanation could be that the size of the $\mathrm{CO}$ envelope in this object is smaller than the photodissociation radius given by the model of Mamon et al. (1988). Decreasing the size of the CO envelope solves the problem with the model producing resolved lines. Also, a smaller radius will require a higher mass-loss rate to reproduce the observed intensities. This also increases the optical depth. However, the dynamical model estimate of the mass-loss rate is $3 \times 10^{-6} M_{\odot} \mathrm{yr}^{-1}$, a little more than a factor of 2 less than the $\mathrm{CO}$ modelling.

\subsubsection{GX Mon}

The dynamical model gives a mass-loss rate estimate $(2 \times$ $10^{-6} M_{\odot} \mathrm{yr}^{-1}$ ) one order of magnitude lower than the $\mathrm{CO}$ model $\left(2 \times 10^{-5} M_{\odot} \mathrm{yr}^{-1}\right)$. The best-fit SED model found according to the strategy described above gives a dust temperature at the inner radius of $500 \mathrm{~K}$. Figure 5 shows the $\chi^{2}$-maps from the SED-fitting and in the case of GX Mon, the model is not very well constrained. The major dust condensation phase in M-type stars is thought to occur at temperatures between $1200 \mathrm{~K}$ and $800 \mathrm{~K}$ (Whittet 2003). With the low dust optical depth derived for GX Mon a high dust-to-gas ratio $(\Psi=0.01)$ is needed to accelerate the gas to the measured expansion velocity. (Setting $T_{\mathrm{d}}\left(r_{\mathrm{i}}\right)=1000 \mathrm{~K}$, requires a higher optical depth to fit the model to the observed fluxes $\left(\tau_{10}=0.85\right)$ and results in $\dot{M}=$ $6.3 \times 10^{-6} M_{\odot} \mathrm{yr}^{-1}$ from the dynamical model, somewhat more in line with the $\mathrm{CO}$ results. The measured expansion velocity would be reached with $\Psi \approx 8.3 \times 10^{-3}$ ). A possible explanation for the low temperature at the inner radius is that the star has undergone a rather drastic decrease in the mass-loss rate recently. The dust envelope from an earlier epoch would have moved outward and cooled to the temperature obtained from the modelling, and the photometric fluxes would thus probe this "older" shell. In such a case the dynamical model would produce an erroneous result.

\subsubsection{WX Psc}

The $J=1 \rightarrow 0,2 \rightarrow 1,3 \rightarrow 2$, and $4 \rightarrow 3$ lines are wellfitted by a constant mass-loss rate of $\dot{M}=4 \times 10^{-5} M_{\odot} \mathrm{yr}^{-1}$. We are, however, not able to fit the $J=6 \rightarrow 5$ line using this model. The problem of not being able to model higherfrequency $\mathrm{CO}$ lines from this source using a constant massloss rate has been previously noted by Kemper et al. (2003),
Teyssier et al. (2006), and Decin et al. (2007). The difficulty of getting well-calibrated data for these higher frequency lines should be kept in mind when drawing conclusions based on this kind of data. As an example, the integrated intensity in the $6 \rightarrow 5$ line in this work is a factor of two stronger than that reported by Kemper et al. (2003). A model fitting only our $6 \rightarrow 5$ line results in $\dot{M}=4 \times 10^{-6} M_{\odot} \mathrm{yr}^{-1}$. Decin et al. (2007) introduce a rather complicated mass-loss history, and estimate the mass-loss rate to be $6 \times 10^{-6} M_{\odot} \mathrm{yr}^{-1}$ in the region where the $6 \rightarrow 5$ line is mainly coming from. Higher transition CO lines are within the range of archived ISO LWS spectra $(43-197 \mu \mathrm{m})$. The CO $J=16 \rightarrow 15$ and $J=18 \rightarrow 17$ lines were modelled using our best-fit model. The resulting peak intensities $\left(4.6 \times 10^{-22} \mathrm{~W} \mathrm{~cm}^{-2}\left(\mathrm{~km} \mathrm{~s}^{-1}\right.\right.$ and $5.0 \times 10^{-22} \mathrm{~W} \mathrm{~cm}^{-2}\left(\mathrm{~km} \mathrm{~s}^{-1}\right.$, respectively) would result in a $S / N \approx 5$ (the resolution-corrected $1 \sigma$ noise level is $\left.8.9 \times 10^{-23} \mathrm{~W} \mathrm{~cm}^{-2}\left(\mathrm{~km} \mathrm{~s}^{-1}\right)\right)$. None of the CO lines within the range of the ISO LWS were detected, and this is in line with the weakness of the observed $J=6 \rightarrow 5$ line. Thus, the results reported by us and previous authors suggest a recent decrease in the mass-loss rate in this source. From the dynamical model we find $\dot{M}=1.8 \times 10^{-5} M_{\odot} \mathrm{yr}^{-1}$.

\subsubsection{IK Tau}

Modelling IK Tau with a constant mass-loss rate of $1 \times$ $10^{-5} M_{\odot} \mathrm{yr}^{-1}$ gives a good fit to the four available CO lines (Fig. C.7). This rate is a factor of two higher than that found by Teyssier et al. (2006) at a somewhat smaller distance of $250 \mathrm{pc}$. The dynamical model gives $\dot{M}=7 \times 10^{-6} M_{\odot} \mathrm{yr}^{-1}$.

\subsubsection{IRC-10529}

When modelling IRC-10529, we encounter a similar problem as for V384 Per. We find that the best-fit CO model with $\dot{M}=$ $3 \times 10^{-5} M_{\odot} \mathrm{yr}^{-1}$ has a $\chi_{\text {red }}^{2}=3.8$. The $J=1 \rightarrow 0$ and $3 \rightarrow 2$ lines are fitted within the observational uncertainties, but the model $2 \rightarrow 1$ line is $45 \%$ too weak, and the $4 \rightarrow 3$ line is $40 \%$ too strong (Fig. C.8). We do not find it likely that this is due to modulations in the mass-loss rate, since the emitting regions for the $1 \rightarrow 0$ and $2 \rightarrow 1$ lines overlap to a large extent. It is more likely due to observational problems. The dynamical model gives $\dot{M}=2.6 \times 10^{-5} M_{\odot} \mathrm{yr}^{-1}$, in good agreement with the estimate from the $\mathrm{CO}$ model.

\section{Discussion}

\subsection{Dependence on parameters}

The model CO line intensities dependence on the $h$-parameter and $\dot{M}$ is displayed in Fig. 2. A further discussion on how the resulting line intensities depend on various input parameters can be found in Schöier \& Olofsson (2001).

To evaluate the sensitivity of the results of the dynamical model to the different derived or assumed input parameters, we varied the parameters in Eqs. (4) and (5) by $\pm 50 \%$. Shown in Table 7 is the needed change of the dust-to-gas ratio, $\Psi$, and the mass-loss rate, $\dot{M}$, to reach the observed expansion velocity and derived optical depth for RW LMi. As can be seen, the dynamical model results are strongly dependent on the assumed dust parameters, which are not well known.

A trend with mass-loss rate can be seen in the dynamical model. For TX Cam, an intermediate-mass-loss-rate object $\left(\dot{M}=3 \times 10^{-6} M_{\odot} \mathrm{yr}^{-1}\right.$ from the dynamical model), an increase in the mass-loss rate by $50 \%$ leads to an increased 
Table 7. The resulting change in mass-loss rate $(\dot{M})$ and dust-to-gas ratio $(\Psi)$ when varying the input parameters in the dynamical model of RW LMi.

\begin{tabular}{clll}
\hline \hline Parameter & Change & $\dot{M}$ & $\Psi$ \\
\hline$L$ & $+50 \%$ & $+50 \%$ & $-35 \%$ \\
\multirow{2}{*}{$M_{*}$} & $-50 \%$ & $-50 \%$ & $+100 \%$ \\
& $+50 \%$ & $-25 \%$ & $+30 \%$ \\
$\sigma_{\mathrm{d}}$ & $-50 \%$ & $+30 \%$ & $-30 \%$ \\
& $+50 \%$ & $+50 \%$ & $-35 \%$ \\
$m_{\mathrm{d}}$ & $-50 \%$ & $-50 \%$ & $+125 \%$ \\
& $+50 \%$ & $-35 \%$ & $+60 \%$ \\
$\tau_{10}$ & $-50 \%$ & $+105 \%$ & $-55 \%$ \\
\multirow{2}{*}{$Q_{\lambda}$} & $+50 \%$ & $+35 \%$ & $+10 \%$ \\
& $-50 \%$ & $-40 \%$ & $-10 \%$ \\
& $+50 \%$ & $+50 \%$ & $-35 \%$ \\
& $-50 \%$ & $-50 \%$ & $+100 \%$ \\
\hline
\end{tabular}

expansion velocity by $6 \%$. For RW LMi, a high-mass-loss-rate object $\left(\dot{M}=1.7 \times 10^{-5} M_{\odot} \mathrm{yr}^{-1}\right)$ the same increase in the massloss rate will lead to a decrease in the expansion velocity by $15 \%$. This is due to the fact that a higher mass-loss rate leads to a higher dust optical depth resulting in a radiation field that decreases more rapidly with radius and with it the radiation pressure efficiency. Nevertheless, this does not lead to a problem finding the correct mass-loss rate. In some cases, two mass-loss rates give the same expansion velocity, but there is only one solution that produces the correct dust optical depth.

We have decided not to display any error bars on the estimates from the dynamical model in Fig. 7. The reason for this is that the resulting (formal) error bars given by the dust radiative transfer model (variations in inner radius and optical depth, Fig. 5) are small and ignores the substantial uncertainties in the adopted dust parameters.

\subsection{Comparing the two methods}

It is very difficult to assess which method is the most reliable for estimating mass-loss rates, since both are dependent on a number of assumptions, which are more or less well-founded. For instance, the dynamical model assumes an entirely dust driven wind, while recent results suggest that there might be other factors at play (Woitke 2006; Höfner \& Andersen 2007).

In all cases but GX Mon, for which we have good reasons to believe that the dynamical model gives an erroneous result (see Sect. 8.2.2 for an explanation), the mass-loss rates estimated from the dynamical model and the CO line modelling agree within a factor of $\sim 3$. A comparison is shown in Fig. 7, where the full drawn line marks the one-to-one relation, while the dashed lines subtend the range within a factor of 3. (Error bars represents the range within the innermost contour in the $\chi^{2}$-maps from the CO model. Here the shape of the line profiles have also been taken into account.) Since the two methods give independent estimates of the mass-loss rate, this result can be used as a guideline to the uncertainty in the mass-lossrate estimates within the adopted circumstellar model. Heras \& Hony (2005) found in their comparison of mass-loss rates derived from ISO-SWS and CO mass-loss rates taken from the literature for low-mass-loss-rate M-type stars that the estimated mass-loss rates can differ up to a factor of ten.

In principle, it is possible to check whether the dust results obtained from the dynamical model $\left(v_{\mathrm{dr}}, \Psi,\left\langle Q_{\mathrm{rp}}\right\rangle^{\dagger}\right)$ are consistent with those used in the $\mathrm{CO}$ radiative transfer modelling $\left(h,\left\langle Q_{\mathrm{rp}}\right\rangle^{\dagger}\right)$. However, we do not expect to get a perfect agreement since in the CO radiative transfer modelling the $h$-parameter is adjusted until the radial kinetic temperature profile results in reasonable relative strengths of the $\mathrm{CO}$ lines. Hence, it is affected by possible missing heating and cooling terms in the description of the thermodynamics of the circumstellar gas. However, as outlined in Sect. 5, this does not affect the mass-loss-rate estimates.

For the carbon stars in our sample the dust-to-gas ratio, $\Psi$, is estimated to be $0.001<\Psi<0.003$. Groenewegen et al. (1998) derived dust-to-gas ratios for a sample of 36 carbon stars and found a rather constant value of 0.0025 up to $P \approx 500$ where a strong increase with period is found. This agrees rather well with our results. However for AFGL 3068 they find $\Psi \approx 0.02$, in strong excess of our result. For the M-type stars we find somewhat larger dust-to-gas ratios, $0.002<\Psi<0.004$ (GX Mon excluded). Justtanont et al. (1994) modelled three thick M-type CSEs and found $0.003<\Psi<0.006$.

The CO mass-loss-rate estimate is lower than the estimate from the dynamical model for all five carbon stars, see Fig. 7. The opposite can be seen for the M-type stars, where the CO model gives a mass-loss-rate estimate higher than the dynamical model for three out of four M-type stars (GX Mon excluded). Keeping in mind that the sample presented here is too small to make a general statement, we suggest that a possible explanation to this is that the adopted $\mathrm{CO} / \mathrm{H}_{2}$-ratio is too low, on average, for the M-type stars and too high for the carbon stars. This is also in line with recent chemical models (Cherchneff 2006), where a non-equilibrium chemical model gives a $\mathrm{CO}$-abundance of $6 \times$ $10^{-4}$ in M-type stars $(\mathrm{C} / \mathrm{O}=0.75)$ at 5 stellar radii. For the carbon stars $(\mathrm{C} / \mathrm{O}=1.1)$, the resulting $\mathrm{CO}$-abundance at the same distance from the star is found to be $9 \times 10^{-4}$. Adopting these abundances would require a higher mass-loss rate for the carbon stars and a lower mass-loss rate for the M-type stars to reproduce the observed $\mathrm{CO}$ line intensities.

\subsection{Comments on some results by Kemper et al. (2003)}

Kemper et al. (2003) made non-LTE radiative transfer modelling of CO $J=2 \rightarrow 1$ to $J=6 \rightarrow 5$ emission toward a sample of AGB stars and red supergiants. In a line ratio diagram they compared modelled line intensity ratios, for standard CSEs with different mass-loss rates, to those found from observations (Fig. 4 in their paper). Kemper et al. found that they could not reproduce the observed line intensity ratios. On the contrary, the standard model described above (Sect. 5) generally gives a very good fit to the observed line intensities as indicated by a $\chi_{\text {red }}^{2}$ of the order of unity for the majority of the sample sources. This is also illustrated in Fig. 8 where line intensity ratios calculated from our standard model using $v_{\mathrm{e}}=15 \mathrm{~km} \mathrm{~s}^{-1}$ and $h=0.5$ for a range of mass-loss rates are compared to the observations. The model CSE was placed at a distance of $1 \mathrm{kpc}$. The interconnected stars are the results from the standard model and the squares show the observed line ratios.

Kemper et al. (2003) also found that the magnitude of the stochastic velocity strongly affects the line intensities, since it determines the size of the region where the line is formed $\left(v_{\text {sto }}=\left(v_{\text {turb }}^{2}+v_{\text {therm }}^{2}\right)^{0.5} \approx v_{\text {turb }}\right.$, since $v_{\text {therm }}$ is the local thermal broadening and $\left.v_{\text {therm }} \ll v_{\text {turb }}\right)$. We have performed the same test as in their Fig. 10, changing the turbulent velocity from 0.05 to $2 \mathrm{~km} \mathrm{~s}^{-1}$, to see how this affects the intensity of the $\mathrm{CO}(J=3 \rightarrow 2)$ line. We used the same standard model as above with a mass-loss rate of $\dot{M}=1 \times 10^{-5} M_{\odot} \mathrm{yr}^{-1}$. Kemper et al. (2003) saw a decrease in intensity from around $T_{\mathrm{mb}}=2.4 \mathrm{~K}$ at $v_{\text {sto }}=0.05 \mathrm{~km} \mathrm{~s}^{-1}$ to $T_{\mathrm{mb}}=0.2 \mathrm{~K}$ at $v_{\text {sto }}=2.0 \mathrm{~km} \mathrm{~s}^{-1}$. 


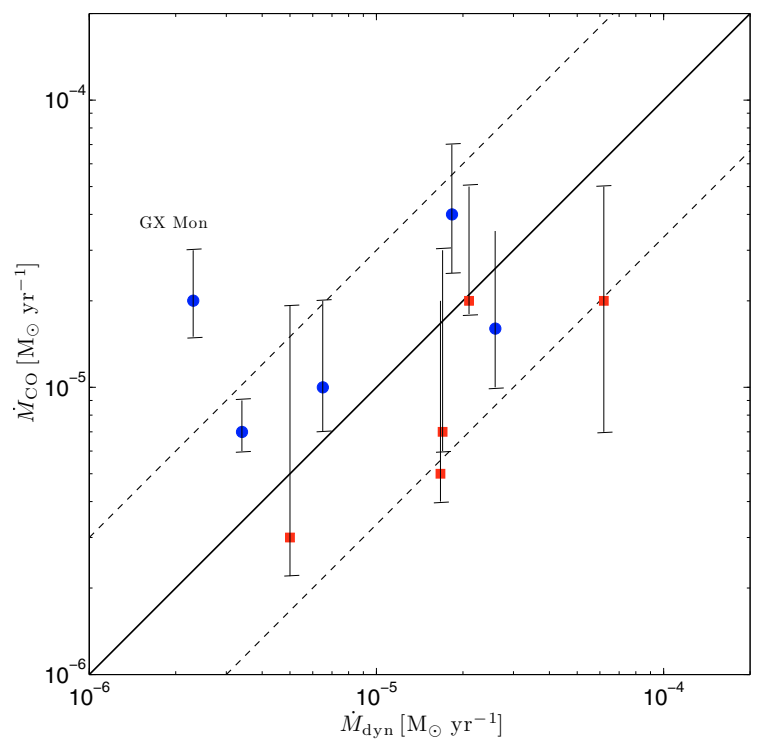

Fig. 7. Comparing the mass-loss-rate results from the $\mathrm{CO}$ model and the dynamical model. Dots mark the M-type stars, and squares the carbon stars. The full drawn line represents a one-to-one relation and the dashed lines subtend the range within a factor of 3 .

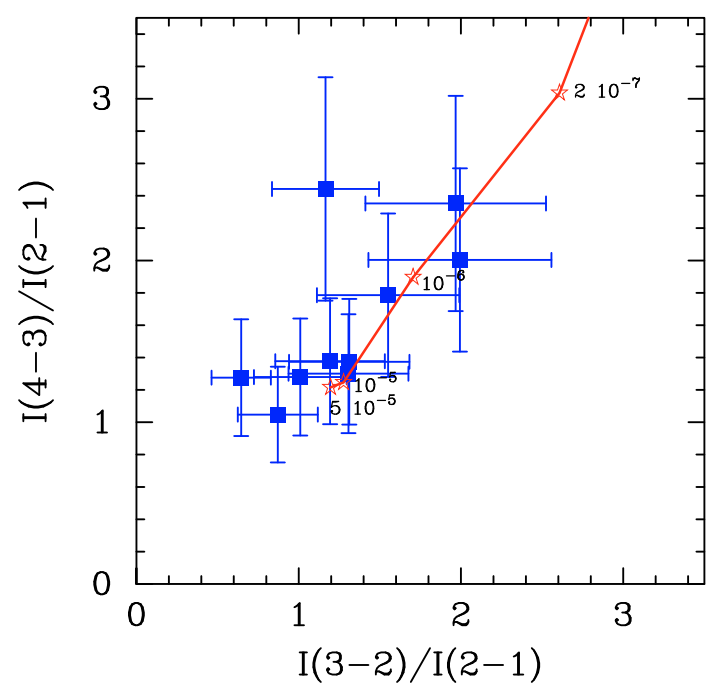

Fig. 8. Observed line intensity ratios for the sample sources (squares) compared with predictions from the standard model for various massloss rates (stars).

We, however, see the opposite trend and a much weaker effect; $T_{\mathrm{mb}}=1.05 \mathrm{~K}$ at $v_{\text {turb }}=0.05 \mathrm{~km} \mathrm{~s}^{-1}$ to $T_{\mathrm{mb}}=1.28 \mathrm{~K}$ at $v_{\text {turb }}=2.0 \mathrm{~km} \mathrm{~s}^{-1}$. It is difficult to outline exactly how the line intensity is affected by the turbulent velocity, as it also affects the excitation of the molecules. However, to a first approximation, with an increased turbulent width the number of molecules per unit velocity interval decreases. On the other hand, as already mentioned above, it also increases the size of the interacting region. Under certain circumstances (spherically symmetric CSE, constant excitation temperature, $v_{\text {turb }} \ll v_{\mathrm{e}}$ ) these two effects cancel out and the line intensity becomes independent of the turbulent velocity (see for example Morris 1975; Olofsson et al. 1982).

In conclusion, we are not able to reproduce the results reported by Kemper et al. (2003) using our model. We confirmed our result using two additional codes; an accelerated Monte Carlo code (Hogerheijde \& van der Tak 2000) and an Accelerated Lambda Iteration (ALI) code (Maercker et al. 2008). The results from the excitation analysis were then combined with different ray tracers (the one used in this paper and the code described in Hogerheijde \& van der Tak 2000) to get the resulting line profiles. All results were in good agreement with ours.

\section{Conclusions}

We have presented mass-loss-rate estimates, derived using two independent methods, for a sample of 10 intermediate- to highmass-loss-rate, well-studied AGB stars, and we arrive at the following conclusions:

- The mass-loss-rate estimates obtained using the two different methods agree within a factor of $\sim 3$, suggesting that this is (at least) the uncertainty in present mass-loss-rate estimates, e.g., using $\mathrm{CO}$ radio line emission. The average of the ratio between the two mass-loss-rate estimates is $\left\langle\dot{M}_{\text {dyn }} / \dot{M}_{\mathrm{CO}}\right\rangle=$ $2.3 \pm 1.0$ for the carbon stars, and $\left\langle\dot{M}_{\mathrm{dyn}} / \dot{M}_{\mathrm{CO}}\right\rangle=0.8 \pm 0.5$ for the M-type stars (excluding GX Mon).

- In most cases, it is not possible to set an upper limit to the mass-loss rate for the high-mass-loss-rate objects by modelling only the integrated $\mathrm{CO}$ line intensities. The analysis can be improved by taking the shape of the line profile into account.

- We are able to successfully model the $\operatorname{CO}(J=1 \rightarrow 0$ to $4 \rightarrow 3$ ) data of all stars using a constant mass-loss rate. However, in most cases there are various indications that our "simple" circumstellar model does not fully reproduce the data. Effects of time-variable mass loss and non-isotropic mass loss may be present.

- We do not find any trends with mass-loss rate when comparing the two models, but we find a possible dependence on chemical type. Generally, the $\mathrm{CO}$ model estimates a lower mass-loss rate for the carbon stars than the dynamical model, while the opposite behaviour can be observed for the M-type stars. We conclude that a $\mathrm{CO} / \mathrm{H}_{2}$-ratio increased and decreased by about a factor of 1.5 for the M- and C-stars, respectively, would give a very good agreement between the methods, but caution that this is a tentative conclusion considering that the sample is small, and there may be other systematic errors.

Acknowledgements. The authors acknowledge support from the Swedish Research Council. This article made use of data obtained through the JCMT archive as Guest User at the Canadian Astronomy Data Center, which is operated by the Dominion Astrophysical Observatory for the National Research Council of Canada's Herzberg Institute of Astrophysics. Finally the authors would like to thank Franz Kerschbaum for providing us with a compilation of near-IR data needed for the dust radiative transfer modelling.

\section{References}

Bagnulo, S. 1996, Ph.D. Thesis, Ph.D. dissertation, Queen's University Belfast, N. Ireland

Bains, I., Cohen, R. J., Louridas, A., et al. 2003, MNRAS, 342, 8

Battinelli, P., \& Demers, S. 2004, A\&A, 418, 33

Beichman, C. A., Neugebauer, G., Habing, H. J., Clegg, P. E., \& Chester, T. J., 1988, Infrared astronomical satellite (IRAS) catalogs and atlases. Explanatory supplement, 1

Bergeat, J., Knapik, A., \& Rutily, B. 2001, A\&A, 369, 178

Bloecker, T. 1995, A\&A, 297, 727

Bowen, G. H. 1988, ApJ, 329, 299

Cherchneff, I. 2006, A\&A, 456, 1001

Cohen, M., Walker, R. G., \& Witteborn, F. C. 1992, AJ, 104, 2030

Decin, L., Hony, S., de Koter, A., et al. 2007, arXiv e-prints, 708 
Dehaes, S., Groenewegen, M. A. T., Decin, L., et al. 2007, MNRAS, 377, 931

Dorschner, J., \& Henning, T. 1995, A\&ARv, 6, 271

Epchtein, N., Matsuura, O. T., Braz, M. A., et al. 1985, A\&AS, 61, 203

Feast, M. W., Whitelock, P. A., \& Menzies, J. W. 2006, MNRAS, 369, 791

Fong, D., Meixner, M., Sutton, E. C., Zalucha, A., \& Welch, W. J. 2006, ApJ, 652,1626

Forestini, M., \& Charbonnel, C. 1997, A\&AS, 123, 241

Gezari, D. Y., Schmitz, M., \& Mead, J. M. 1987, Catalog of infrared observations. Part 1: Data, Tech. rep.

González Delgado, D., Olofsson, H., Kerschbaum, F., et al. 2003, A\&A, 411, 123

Groenewegen, M. A. T. 1997, A\&A, 317, 503

Groenewegen, M. A. T., de Jong, T., van der Bliek, N. S., Slijkhuis, S., \& Willems, F. J. 1992, A\&A, 253, 150

Groenewegen, M. A. T., de Jong, T., \& Baas, F. 1993, A\&AS, 101, 513

Groenewegen, M. A. T., van der Veen, W. E. C. J., \& Matthews, H. E. 1998, A\&A, 338, 491

Habing, H. J. 1996, A\&ARv, 7, 97

Habing, H. J., Tignon, J., \& Tielens, A. G. G. M. 1994, A\&A, 286, 523

Heras, A. M., \& Hony, S. 2005, A\&A, 439, 171

Heske, A., Forveille, T., Omont, A., van der Veen, W. E. C. J., \& Habing, H. J. 1990, A\&A, 239, 173

Höfner, S., \& Andersen, A. C. 2007, A\&A, 465, L39

Höfner, S., Feuchtinger, M. U., \& Dorfi, E. A. 1995, A\&A, 297, 815

Hogerheijde, M. R., \& van der Tak, F. F. S. 2000, A\&A, 362, 697

Ivezic, Z., \& Elitzur, M. 1997, MNRAS, 287, 799

Jones, T. J., Bryja, C. O., Gehrz, R. D., et al. 1990, ApJS, 74, 785

Justtanont, K., \& Tielens, A. G. G. M. 1992, ApJ, 389, 400

Justtanont, K., Skinner, C. J., \& Tielens, A. G. G. M. 1994, ApJ, 435, 852

Justtanont, K., Skinner, C. J., Tielens, A. G. G. M., Meixner, M., \& Baas, F. 1996, ApJ, 456, 337

Kahane, C., \& Jura, M. 1994, A\&A, 290, 183

Kahane, C., Dufour, E., Busso, M., et al. 2000, A\&A, 357, 669

Karakas, A. I., \& Lattanzio, J. C. 2007, arXiv e-prints, 708

Kastner, J. H. 1992, ApJ, 401, 337

Kemper, F., Stark, R., Justtanont, K., et al. 2003, A\&A, 407, 609

Kerschbaum, F., \& Hron, J. 1994, A\&AS, 106, 397

Knapp, G. R., \& Morris, M. 1985, ApJ, 292, 640

Knapp, G. R., Young, K., Lee, E., \& Jorissen, A. 1998, ApJS, 117, 209

Lambert, D. L., Gustafsson, B., Eriksson, K., \& Hinkle, K. H. 1986, ApJS, 62, 373

Lamers, H. J. G. L. M., \& Cassinelli, J. P. 1999, Introduction to Stellar Winds ed. J. G. L. M. Henny Lamers, \& J. P. Cassinelli (Cambridge, UK: Cambridge University Press), 452

Leão, I. C., de Laverny, P., Mékarnia, D., de Medeiros, J. R., \& Vandame, B. 2006, A\&A, 455, 187

Le Bertre, T. 1992, A\&AS, 94, 377

Le Bertre, T. 1993, A\&AS, 97, 729

Lepine, J. R. D., Ortiz, R., \& Epchtein, N. 1995, A\&A, 299, 453

Maercker, M., Schöier, F. L., Olofsson, H., Bergman, P., \& Ramstedt, S. 2008, A\&A, 479, 779

Mamon, G. A., Glassgold, A. E., \& Huggins, P. J. 1988, ApJ, 328, 797

Marengo, M., Busso, M., Silvestro, G., Persi, P., \& Lagage, P. O. 1999, A\&A, 348,501
Marigo, P., \& Girardi, L. 2007, A\&A, 469, 239

Marshall, C. R., Leahy, D. A., \& Kwok, S. 1992, PASP, 104, 397

Mauron, N., \& Huggins, P. J. 1999, A\&A, 349, 203

Mauron, N., \& Huggins, P. J. 2000, A\&A, 359, 707

Mauron, N., \& Huggins, P. J. 2001, in Galaxies and their Constituents at the

Highest Angular Resolutions, ed. R. T. Schilizzi, IAU Symp., 205, 310

Mauron, N., \& Huggins, P. J. 2006, A\&A, 452, 257

Men'shchikov, A. B., Balega, Y. Y., Berger, M., et al. 2006, A\&A, 448, 271

Monnier, J. D., Geballe, T. R., \& Danchi, W. C. 1998, ApJ, 502, 833

Morris, M. 1975, ApJ, 197, 603

Neri, R., Kahane, C., Lucas, R., Bujarrabal, V., \& Loup, C. 1998, A\&AS, 130, 1

Olivier, E. A., Whitelock, P., \& Marang, F. 2001, MNRAS, 326, 490

Olofsson, H., Johansson, L. E. B., Hjalmarson, A., \& Nguyen-Quang-Rieu 1982, A\&A, 107, 128

Olofsson, H., Eriksson, K., Gustafsson, B., \& Carlstrom, U. 1993, ApJS, 87, 267

Olofsson, H., Bergman, P., Lucas, R., et al. 2000, A\&A, 353, 583

Olofsson, H., González Delgado, D., Kerschbaum, F., \& Schöier, F. L. 2002, A\&A, 391, 1053

Ramstedt, S., Schöier, F. L., Olofsson, H., \& Lundgren, A. A. 2006, A\&A, 454, L103

Sahai, R. 1990, ApJ, 362, 652

Samus, N. N., Durlevich, O. V., et al. 2004, VizieR Online Data Catalog, 2250, 0

Sandin, C., \& Höfner, S. 2003, A\&A, 398, 253

Schöier, F. L., \& Olofsson, H. 2001, A\&A, 368, 969

Schöier, F. L., Ryde, N., \& Olofsson, H. 2002, A\&A, 391, 577

Schöier, F. L., Lindqvist, M., \& Olofsson, H. 2005a, A\&A, 436, 633

Schöier, F. L., van der Tak, F. F. S., van Dishoeck, E. F., \& Black, J. H. 2005b, A\&A, 432, 369

Schröder, K.-P., \& Sedlmayr, E. 2001, A\&A, 366, 913

Sedlmayr, E. 1994, in Molecules in the Stellar Environment, IAU Colloq. 146,

ed. U. G. Jorgensen (Berlin: Springer Verlag), Lect. Notes in Phys., 428, 163

Simis, Y. J. W., Icke, V., \& Dominik, C. 2001, A\&A, 371, 205

Skinner, C. J., Meixner, M., Barlow, M. J., et al. 1997, A\&A, 328, 290

Sopka, R. J., Hildebrand, R., Jaffe, D. T., et al. 1985, ApJ, 294, 242

Speck, A. K., Barlow, M. J., \& Skinner, C. J. 1997, MNRAS, 288, 431

Stanek, K. Z., Knapp, G. R., Young, K., \& Phillips, T. G. 1995, ApJS, 100, 169

Steffen, M., \& Schönberner, D. 2000, A\&A, 357, 180

Suh, K.-W. 2000, MNRAS, 315, 740

Teyssier, D., Hernandez, R., Bujarrabal, V., Yoshida, H., \& Phillips, T. G. 2006,

A\&A, 450, 167

van Loon, J. T. 2006, arXiv Astrophysics e-prints

Volk, K., \& Cohen, M. 1989, AJ, 98, 1918

Walmsley, C. M., Chini, R., Kreysa, E., et al. 1991, A\&A, 248, 555

Whitelock, P., Menzies, J., Feast, M., et al. 1994, MNRAS, 267, 711

Whittet, D. C. B., 2003, Dust in the galactic environment

Willson, L. A. 2000, ARA\&A, 38, 573

Winters, J. M., Le Bertre, T., Jeong, K. S., Nyman, L.-Å., \& Epchtein, N. 2003, A\&A, 409, 715

Woitke, P. 2006, A\&A, 460, L9

Woods, P. M., Schöier, F. L., Nyman, L.-Å., \& Olofsson, H. 2003, A\&A, 402, 617

Zuckerman, B., \& Dyck, H. M. 1986, ApJ, 304, 394 


\section{Appendix A: A new mass loss rate formula}

Knapp \& Morris (1985) derived a theoretical mass-loss-rate formula in terms of the $\mathrm{CO}(J=1 \rightarrow 0)$ line intensity, valid for optically thick CO emission. The formula relates the mass-loss rate to a number of easily determined observables; $T_{\mathrm{mb}}$ for the $\mathrm{CO}(1-0)$ line, and $v_{\mathrm{e}}$. The distance $\mathrm{D}$ and $\mathrm{CO}$ abundance $f_{\mathrm{CO}}$ also enter the formula. It was derived using a fixed $\mathrm{CO}$ envelope size. This formula was used in e.g. Olofsson et al. (1993) to estimate mass-loss rates for a sample of carbon stars. Later, Schöier \& Olofsson (2001) could compare their results on the sample, derived using the same radiative transfer model as in this work, to those estimated using the formula. They found that the formula underestimated the mass-loss rates compared to what was found from the radiative transfer analysis, on average by a factor of four, but the discrepancy was found to increase even more for lower mass-loss-rate objects. The reason is that the emission from the low-mass-loss-rate objects is not optically thick and it is more sensitive to the size of the $\mathrm{CO}$ envelope. However, the usefulness of simple formulae of this sort can not be disputed.

In order to improve the results by Knapp \& Morris (1985), we set up a grid with 60 model stars, whose properties were varied in $\dot{M}\left(1,3,10,30\right.$, and $\left.100 \times 10^{-7} M_{\odot} \mathrm{yr}^{-1}\right), v_{\mathrm{e}}(5,10,15$, and $\left.20 \mathrm{~km} \mathrm{~s}^{-1}\right)$, and $f_{\mathrm{CO}}\left(1,3\right.$, and $\left.10 \times 10^{-4}\right)$. Following Schöier $\&$ Olofsson (2001), the value of $h$ was assumed to be 0.2 for $\dot{M}$ in the range up to $3 \times 10^{-7} M_{\odot} \mathrm{yr}^{-1}, 0.5$ for $1-3 \times 10^{-6} M_{\odot} \mathrm{yr}^{-1}$, and 1.5 for the mass-loss rate $1 \times 10^{-5} M_{\odot} \mathrm{yr}^{-1}$. For each star, the velocity integrated intensities in $\mathrm{CO}(J=1 \rightarrow 0, J=2 \rightarrow 1$, $J=3 \rightarrow 2, J=4 \rightarrow 3$ ) were calculated for a $20 \mathrm{~m}$ class telescope (the corresponding beam sizes were $33^{\prime \prime}, 16.5^{\prime \prime}, 11^{\prime \prime}$, and $\left.8.5^{\prime \prime}\right)$. The distance to the stars were set to $1 \mathrm{kpc}$. The output from the modeling was fitted to Eq. (A.1) and the errors were minimized using a Levenberg-Marquardt method (Press et al. 1992),

$\dot{M}=s_{J}\left(I_{\mathrm{CO}} \theta_{\mathrm{b}}^{2} D^{2}\right)^{a_{J}} v_{\mathrm{e}}^{b_{J}} f_{\mathrm{CO}}^{-c_{J}}$.

Table A.1 summarizes the results. For low upper- $J$ levels, the mass-loss rate is about equally (and moderately) dependent on changes of $I, v_{\mathrm{e}}$, and $f_{\mathrm{CO}}$. At high upper- $J$ levels, the mass-loss rate is almost linearly dependent on the velocity-integrated intensity $(I)$, but relatively insensitive to changes in $v_{\mathrm{e}}$, and $f_{\mathrm{CO}}$. In general, the parameter most difficult to fit was the exponent for the expansion velocity $\left(v_{\mathrm{e}}\right)$, while the exponent for the velocityintegrated intensity $(I)$ is well determined. Equation (A.1) only applies to unresolved emission. Figure A.1 shows a comparison between mass-loss rates estimated using Eq. (A.1) on the $\mathrm{y}$-axis and mass-loss rates estimated using the Monte-Carlo radiative transfer model on the $\mathrm{x}$-axis for three samples of C-, M-, and S-stars (Schöier \& Olofsson 2001; Olofsson et al. 2002; González Delgado et al. 2003; Ramstedt et al. 2006). $f_{\text {CO }}$ is assumed to be $1 \times 10^{-3}$ for the C-stars, $2 \times 10^{-4}$ for the M-stars, and $6 \times 10^{-4}$ for the S-stars.
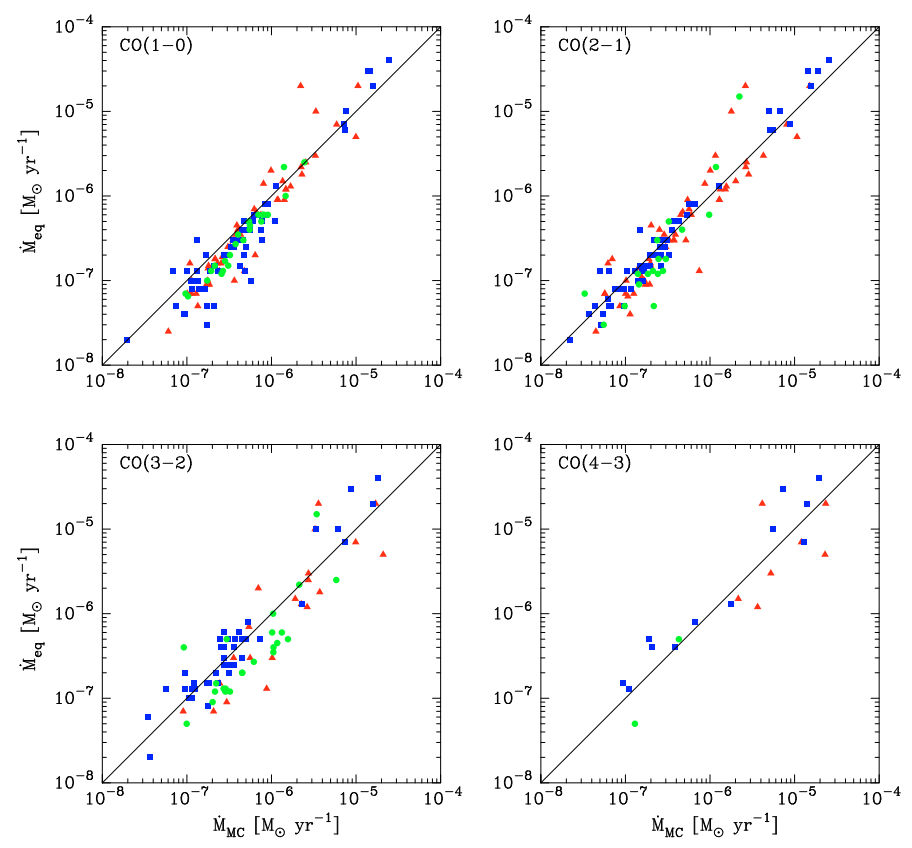

Fig. A.1. Mass-loss rates derived using Eq. (A.1) and the parameters in Table A.1 versus the mass-loss rates estimated using the Monte Carlo model for the same stars. Red triangles represent carbon stars, blue squares, M-type stars, and green dots represent S-type stars.

Table A.1. Values for the parameters in Eq. (A.1). $s_{J}$ is calibrated such that Eq. (A.1) gives the mass-loss rate in $M_{\odot} \mathrm{yr}^{-1}$. Values are valid in the ranges $10^{-7} \leq \dot{M} \leq 10^{-5} M_{\odot} \mathrm{yr}^{-1}, 5 \leq v_{\mathrm{e}} \leq 20 \mathrm{~km} \mathrm{~s}^{-1}, 10^{-4} \leq f_{\mathrm{CO}} \leq$ $10^{-3}$.

\begin{tabular}{lcccc}
\hline \hline Transition & $s_{J}$ & $a_{J}(I)$ & $b_{J}\left(v_{\mathrm{e}}\right)$ & $c_{J}\left(f_{\mathrm{CO}}\right)$ \\
\hline$J=1 \rightarrow 0$ & $4.9( \pm 2.4) \times 10^{-12}$ & $0.68( \pm 0.03)$ & $0.59( \pm 0.13)$ & $0.80( \pm 0.08)$ \\
$J=2 \rightarrow 1$ & $1.3( \pm 0.7) \times 10^{-11}$ & $0.82( \pm 0.03)$ & $0.46( \pm 0.13)$ & $0.59( \pm 0.07)$ \\
$J=3 \rightarrow 2$ & $3.8( \pm 3.4) \times 10^{-11}$ & $0.91( \pm 0.04)$ & $0.39( \pm 0.13)$ & $0.45( \pm 0.07)$ \\
$J=4 \rightarrow 3$ & $8.4( \pm 4.1) \times 10^{-11}$ & $0.95( \pm 0.04)$ & $0.36( \pm 0.13)$ & $0.37( \pm 0.07)$ \\
\hline
\end{tabular}


Table B.1. Photometric flux densities in $10^{-26} \mathrm{~W} \mathrm{~m}^{-2} \mathrm{~Hz}^{-1}$.

\begin{tabular}{|c|c|c|c|c|c|c|c|c|c|c|}
\hline $\begin{array}{l}\text { Wavelength } \\
{[\mu \mathrm{m}]}\end{array}$ & $\overline{\text { LP And }}$ & CW Leo & V384 Per & AFGL 3068 & $\overline{\text { RW LMi }}$ & TX Cam & GX Mon & $\overline{\text { WX Psc }}$ & $\overline{\mathrm{IK}}$ Tau & IRC-10529 \\
\hline 1.24 & $1.6^{a}$ & $4.0^{d}$ & $13^{e}$ & $\cdots$ & $5.8^{h}$ & $280^{a}$ & $49^{J}$ & $3.2^{i}$ & $250^{t}$ & $2.4^{l}$ \\
\hline 1.63 & $11^{a}$ & $58^{d}$ & $53^{e}$ & $\ldots$ & $39^{h}$ & $590^{a}$ & $120^{j}$ & $32^{i}$ & $730^{i}$ & $20^{i}$ \\
\hline 2.19 & $55^{a}$ & $520^{d}$ & $170^{e}$ & $\ldots$ & $180^{h}$ & $780^{a}$ & $200^{j}$ & $130^{i}$ & $1200^{i}$ & $78^{i}$ \\
\hline 3.79 & $450^{a}$ & $10000^{d}$ & $520^{e}$ & $9.6^{g}$ & $970^{h}$ & $750^{a}$ & $580^{j}$ & $620^{i}$ & $1900^{i}$ & $290^{i}$ \\
\hline 4.64 & $\ldots$ & $19000^{d}$ & $\ldots$ & $55^{g}$ & $\ldots$ & $\ldots$ & $410^{j}$ & $800^{i}$ & $1800^{i}$ & $340^{i}$ \\
\hline 8.55 & $\ldots$ & $\ldots$ & $\ldots$ & $290^{k}$ & $\ldots$ & $\ldots$ & $\ldots$ & $850^{k}$ & $\ldots$ & $\ldots$ \\
\hline 8.57 & $\ldots$ & $\ldots$ & $\ldots$ & $\ldots$ & $\ldots$ & $\ldots$ & $\ldots$ & $700^{k}$ & $\ldots$ & $\ldots$ \\
\hline 9.56 & $\ldots$ & $\ldots$ & $\ldots$ & $360^{k}$ & $\ldots$ & $\ldots$ & $\ldots$ & $1100^{k}$ & $\ldots$ & $\ldots$ \\
\hline 11.21 & $\ldots$ & $\ldots$ & $\ldots$ & $400^{k}$ & $3900^{k}$ & $\ldots$ & $\ldots$ & $1000^{k}$ & $\ldots$ & $\ldots$ \\
\hline 12 & $960^{b}$ & $48000^{b}$ & $540^{b}$ & $1700^{b}$ & $3300^{b}$ & $1600^{b}$ & $1600^{b}$ & $1200^{b}$ & $4600^{b}$ & $1300^{b}$ \\
\hline 12.45 & $\ldots$ & $\ldots$ & $\ldots$ & $470^{k}$ & $\ldots$ & $\ldots$ & $\ldots$ & $360^{k}$ & $\ldots$ & $\ldots$ \\
\hline 25 & $470^{b}$ & $23000^{b}$ & $200^{b}$ & $780^{b}$ & $1200^{b}$ & $640^{b}$ & $360^{b}$ & $970^{b}$ & $2400^{b}$ & $1100^{b}$ \\
\hline 60 & $110^{b}$ & $5700^{b}$ & $40^{b}$ & $250^{b}$ & $270^{b}$ & $130^{b}$ & $110^{b}$ & $220^{b}$ & $330^{b}$ & $220^{b}$ \\
\hline 100 & $36^{b}$ & $920^{b}$ & $12^{b}$ & $74^{b}$ & $86^{b}$ & $39^{b}$ & $40^{b}$ & $72^{b}$ & $100^{b}$ & $64^{b}$ \\
\hline 400 & $\ldots$ & $\ldots$ & $\cdots$ & $\ldots$ & $\ldots$ & $\cdots$ & $\ldots$ & $3.0^{m}$ & $\ldots$ & $\ldots$ \\
\hline 450 & $0.35^{f \dagger}$ & $13^{\circ}$ & $\ldots$ & $2.2^{\circ}$ & $2.4^{o}$ & $\ldots$ & $\ldots$ & $\ldots$ & $1.2^{l \dagger}$ & $\ldots$ \\
\hline 600 & $0.41^{f}$ & $\ldots$ & $0.11^{f \dagger}$ & $0.67^{f}$ & $\ldots$ & $\ldots$ & $\ldots$ & $\ldots$ & $\ldots$ & $\ldots$ \\
\hline 800 & $0.25^{f}$ & $6.7^{l}$ & $0.21^{f}$ & $0.33^{f}$ & $0.78^{l}$ & $\ldots$ & $\ldots$ & $\ldots$ & $0.25^{l}$ & $\ldots$ \\
\hline 850 & $\ldots$ & $12^{\circ}$ & $\ldots$ & $0.59^{\circ}$ & $0.97^{\circ}$ & $\ldots$ & $\ldots$ & $\ldots$ & $\ldots$ & $\ldots$ \\
\hline 1100 & $0.081^{f}$ & $1.9^{l}$ & $0.045^{f}$ & $0.17^{f}$ & $0.16^{l}$ & $\ldots$ & $\ldots$ & $\ldots$ & $0.082^{l}$ & $\ldots$ \\
\hline 1200 & $\ldots$ & $\ldots$ & $\ldots$ & $\ldots$ & $\ldots$ & $\ldots$ & $\ldots$ & $0.24^{c}$ & $\ldots$ & $0.10^{c}$ \\
\hline 1300 & $\ldots$ & $1.5^{n}$ & $\ldots$ & $\ldots$ & $0.29^{n}$ & $\ldots$ & $\ldots$ & $0.059^{c}$ & $0.037^{n}$ & $\ldots$ \\
\hline
\end{tabular}

References:

${ }^{a}$ Bagnulo (1996); ${ }^{b}$ Beichman et al. (1988), IRAS Point Source Catalogue; ${ }^{c}$ Dehaes et al. (2007); ${ }^{d}$ Epchtein et al. (1985); ${ }^{e}$ Gezari et al. (1987); ${ }^{f}$ Groenewegen et al. (1993); ${ }^{g}$ Jones et al. (1990); ${ }^{h}$ Kerschbaum \& Hron (1994); ${ }^{i}$ Le Bertre (1993); ${ }^{j}$ Lepine et al. (1995); ${ }^{k}$ Marengo et al. (1999); ${ }^{l}$ Marshall et al. (1992); ${ }^{m}$ Sopka et al. (1985); ${ }^{n}$ Walmsley et al. (1991); ${ }^{o}$ JCMT archival data.

Table C.1. The difference between the integrated $\mathrm{CO}(J \rightarrow J-1)$ intensities from the best-fit model and the observations, in percent; $\left(I_{\mathrm{mod}}-I_{\mathrm{obs}}\right) / I_{\mathrm{obs}}$.

\begin{tabular}{|c|c|c|c|c|c|}
\hline \multirow[t]{2}{*}{ Source } & \multirow{2}{*}{$\begin{array}{c}\text { OSO } \\
1 \rightarrow 0\end{array}$} & \multicolumn{3}{|c|}{ JCMT } & \multirow[b]{2}{*}{$6 \rightarrow 5$} \\
\hline & & $2 \rightarrow 1$ & $3 \rightarrow 2$ & $4 \rightarrow 3$ & \\
\hline LP And & 3.2 & 5.4 & 15.8 & 4.2 & -0.6 \\
\hline CW Leo & 4.8 & -7.3 & -15.6 & -11.7 & $\cdots$ \\
\hline RW LMi & -16.6 & 37.4 & -1.6 & 6.2 & $\ldots$ \\
\hline V384 Per & -22.3 & -20.3 & 63.8 & -15.3 & $\cdots$ \\
\hline AFGL 3068 & 6.3 & -4.2 & 11.0 & -13.3 & 34.6 \\
\hline TX Cam & -7.7 & 56.9 & 40.2 & -18.9 & $\ldots$ \\
\hline GX Mon & 1.6 & -3.4 & -1.9 & 3.4 & $\ldots$ \\
\hline WX Psc & -3.2 & -14.8 & 23.8 & 6.8 & 273.7 \\
\hline IK Tau & -3.3 & -1.4 & 9.2 & 16.4 & $\ldots$ \\
\hline IRC-10529 & -3.5 & -44.8 & 11.9 & 38.9 & $\cdots$ \\
\hline
\end{tabular}

\section{Appendix B: Photometric fluxes and dust radiative transfer}

The SEDs used as constraints in the dust emission modelling are constructed from $J H K L M$-band, sub-millimetre, and millimetre photometric flux densities from the literature, together with IRAS data. All flux densities used are listed in Table B.1. Values in italics were omitted from the analysis to avoid a bias in the $\chi^{2}$-analysis at certain wavelengths. Values in italics marked with a dagger were omitted since the reported errors were $\gtrsim 50 \%$.

\section{Appendix C: Results from the $\mathrm{CO}$ line radiative transfer model}

Figures C.1-C.8 show the best-fit models from the CO radiative transfer modelling overlayed the new observational data for all stars in the sample (except LP And and TX Cam shown in Figs. 3 and 4, respectively). The results are discussed in Sect. 8. Table C.1 gives the difference between in integrated intensity from the model and the observation, for each line. 
S. Ramstedt et al.: On the reliability of mass-loss-rate estimates for AGB stars, Online Material p 3
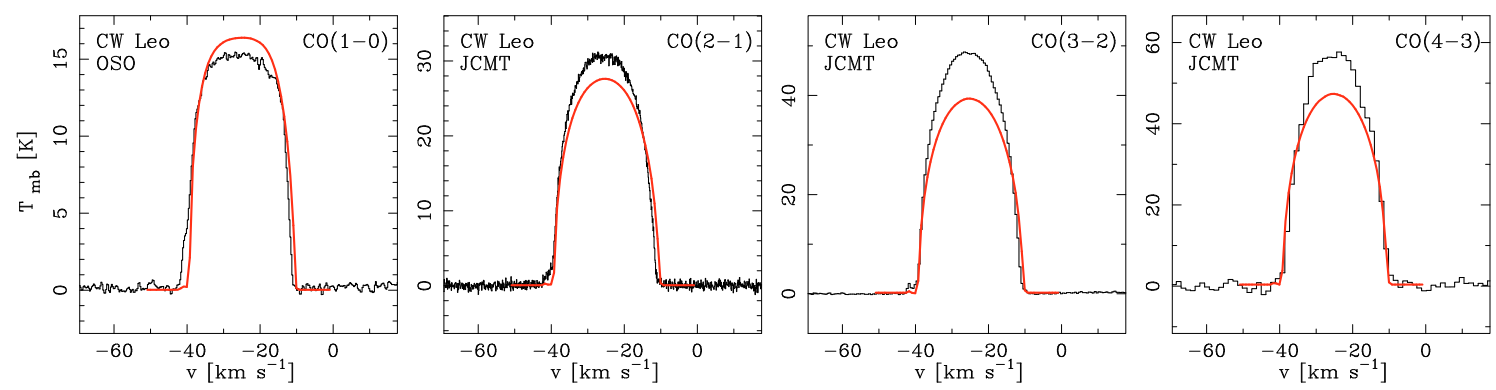

Fig. C.1. The observed CO $J=1 \rightarrow 0$ line from OSO, $2 \rightarrow 1,3 \rightarrow 2$, and $4 \rightarrow 3$ lines from JCMT, of the carbon star CW Leo, overlayed with the best-fit model $\left(\dot{M}=2 \times 10^{-5} M_{\odot} \mathrm{yr}^{-1}\right)$ from the CO radiative transfer modelling. The reduced $\chi^{2}$ for this model is $\chi_{\text {red }}^{2}=0.6$. See Sect. 8.1.2.
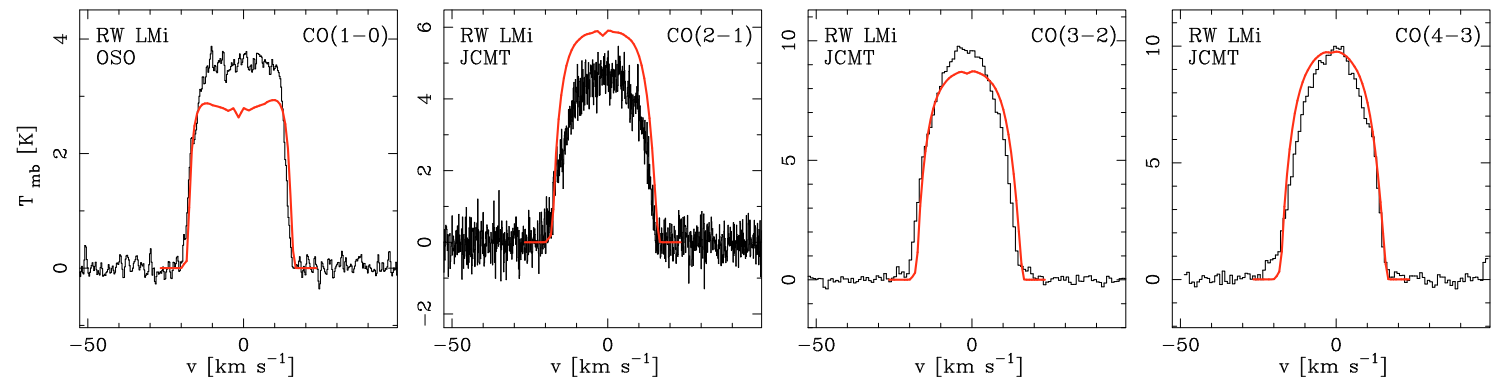

Fig. C.2. The observed CO $J=1 \rightarrow 0$ line from OSO, $2 \rightarrow 1,3 \rightarrow 2$, and $4 \rightarrow 3$ lines from JCMT, of the carbon star RW LMi, overlayed with the best-fit model $\left(\dot{M}=5 \times 10^{-6} M_{\odot} \mathrm{yr}^{-1}\right)$ from the CO radiative transfer modelling. The reduced $\chi^{2}$ for this model is $\chi_{\text {red }}^{2}=2.1$. See Sect. 8.1.5.
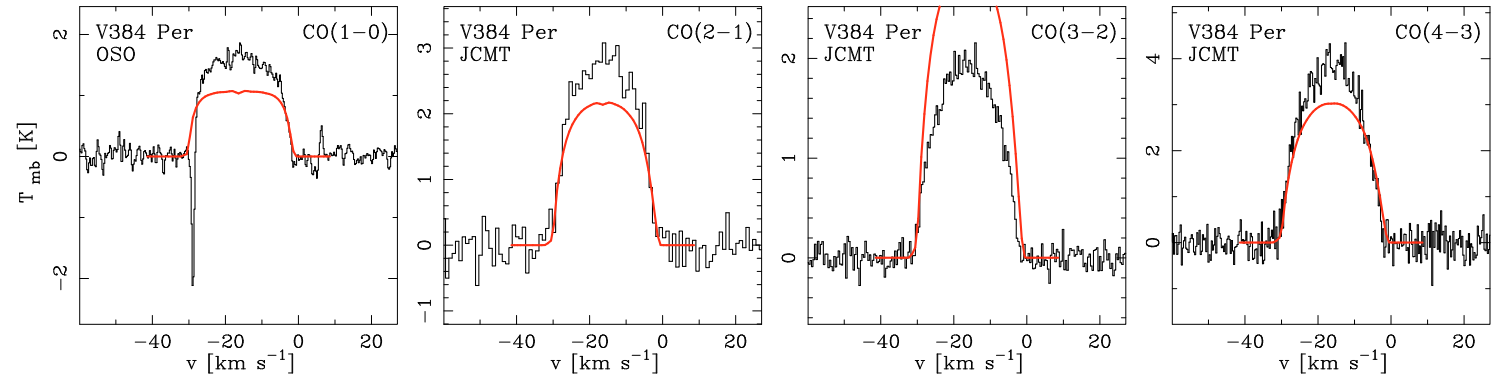

Fig. C.3. The observed CO $J=1 \rightarrow 0$ line from OSO, $2 \rightarrow 1,3 \rightarrow 2$, and $4 \rightarrow 3$ lines from JCMT, of the carbon star V384 Per, overlayed with the best-fit model $\left(\dot{M}=3 \times 10^{-6} M_{\odot} \mathrm{yr}^{-1}\right)$ from the CO radiative transfer modelling. The reduced $\chi^{2}$ for this model is $\chi_{\text {red }}^{2}=3.7$. See Sect. 8.1.3.

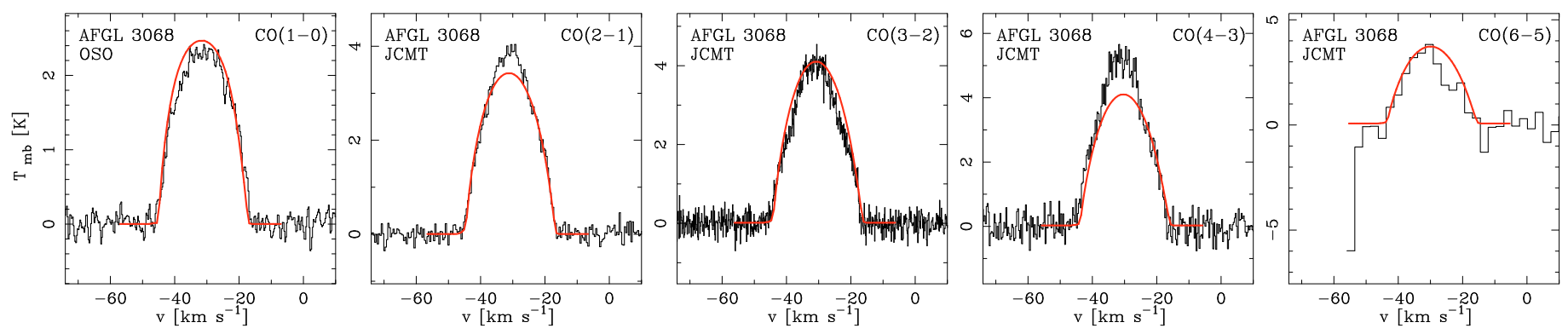

Fig. C.4. The observed CO $J=1 \rightarrow 0$ line from OSO, $2 \rightarrow 1,3 \rightarrow 2,4 \rightarrow 3$, and $6 \rightarrow 5$ lines from JCMT, of the carbon star AFGL 3068 , overlayed with the best-fit model $\left(\dot{M}=2 \times 10^{-5} M_{\odot} \mathrm{yr}^{-1}\right)$ from the CO radiative transfer modelling. The reduced $\chi^{2}$ for this model is $\chi_{\text {red }}^{2}=0.7$. See Sect. 8.1.4. 

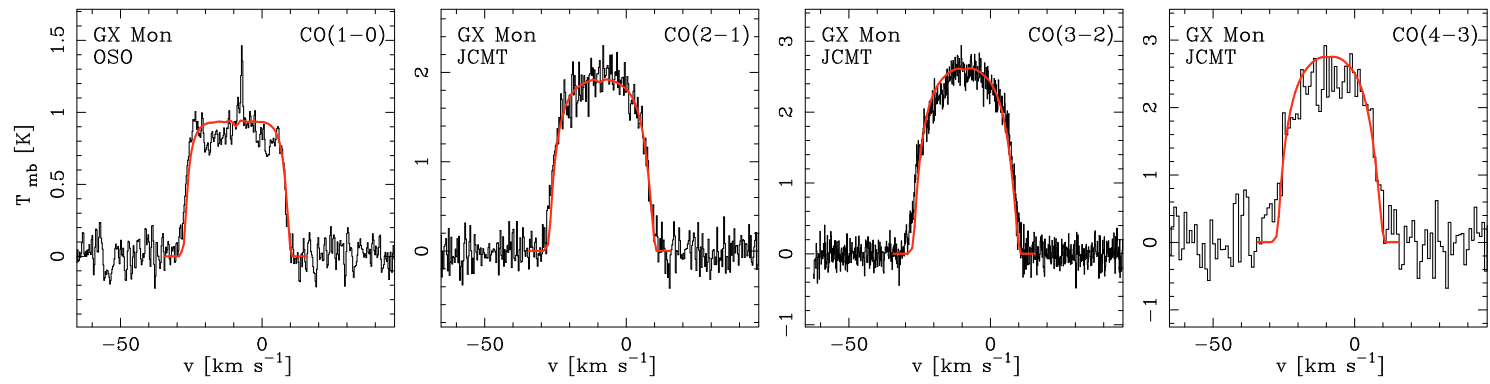

Fig. C.5. The observed CO $J=1 \rightarrow 0$ line from OSO, $2 \rightarrow 1,3 \rightarrow 2$, and $4 \rightarrow 3$ lines from JCMT, of the M-type star GX Mon, overlayed with the best-fit model $\left(\dot{M}=2 \times 10^{-5} M_{\odot} \mathrm{yr}^{-1}\right)$ from the CO radiative transfer modelling. The reduced $\chi^{2}$ for this model is $\chi_{\text {red }}^{2}=0.04$. See Sect. 8.2.2.

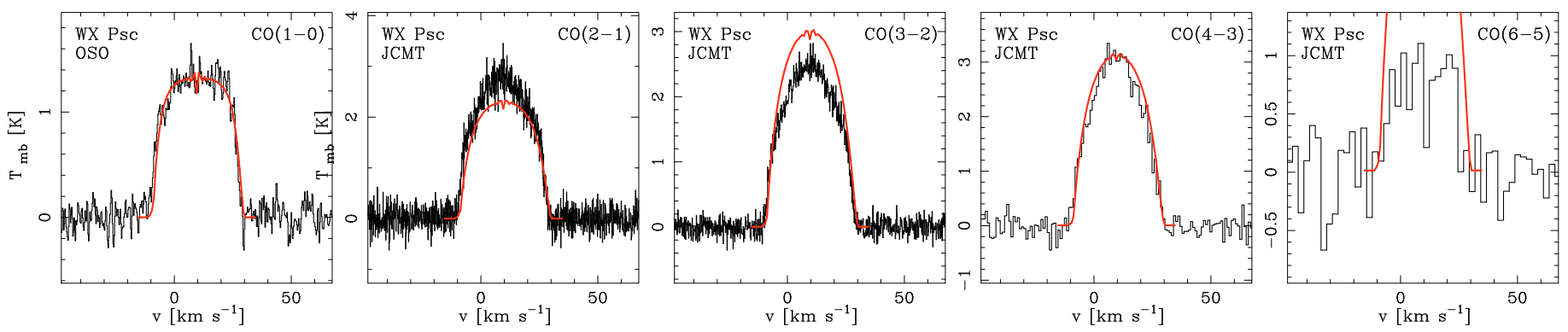

Fig. C.6. The observed CO $J=1 \rightarrow 0$ line from OSO, $2 \rightarrow 1,3 \rightarrow 2,4 \rightarrow 3$, and $6 \rightarrow 5$ lines from JCMT, of the M-type star WX Psc, overlayed with the best-fit model $\left(\dot{M}=4 \times 10^{-5} M_{\odot} \mathrm{yr}^{-1}\right)$ from the CO radiative transfer modelling. The reduced $\chi^{2}$ for this model is $\chi_{\text {red }}^{2}=1.0$, when the $6 \rightarrow 5$ line is omitted. See Sect. 8.2.3.
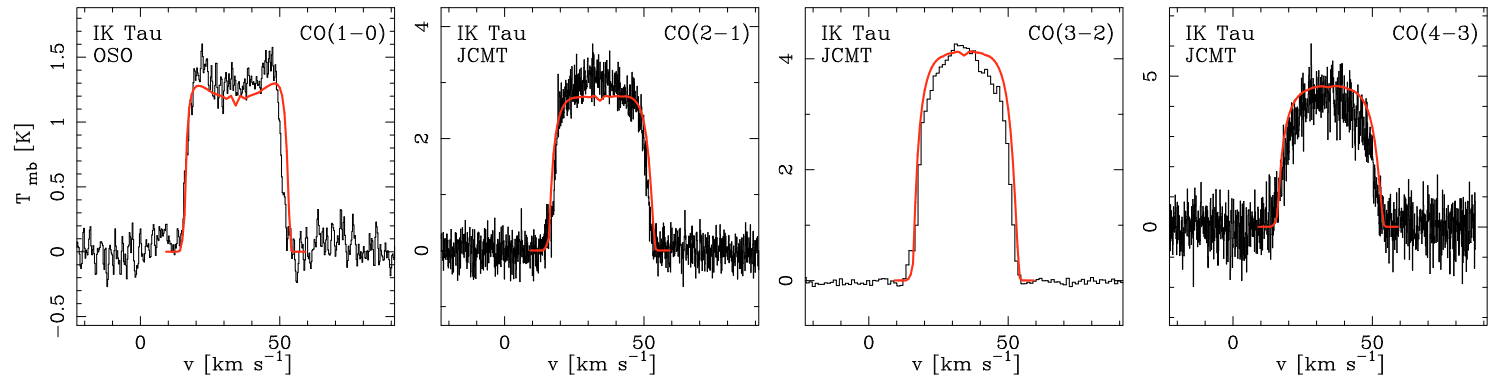

Fig. C.7. The observed CO $J=1 \rightarrow 0$ line from OSO, $2 \rightarrow 1,3 \rightarrow 2$, and $4 \rightarrow 3$ lines from JCMT, of the M-type star IK Tau, overlayed with the best-fit model $\left(\dot{M}=1 \times 10^{-5} M_{\odot} \mathrm{yr}^{-1}\right)$ from the CO radiative transfer modelling. The reduced $\chi^{2}$ for this model is $\chi_{\text {red }}^{2}=0.5$. See Sect. 8.2.4.
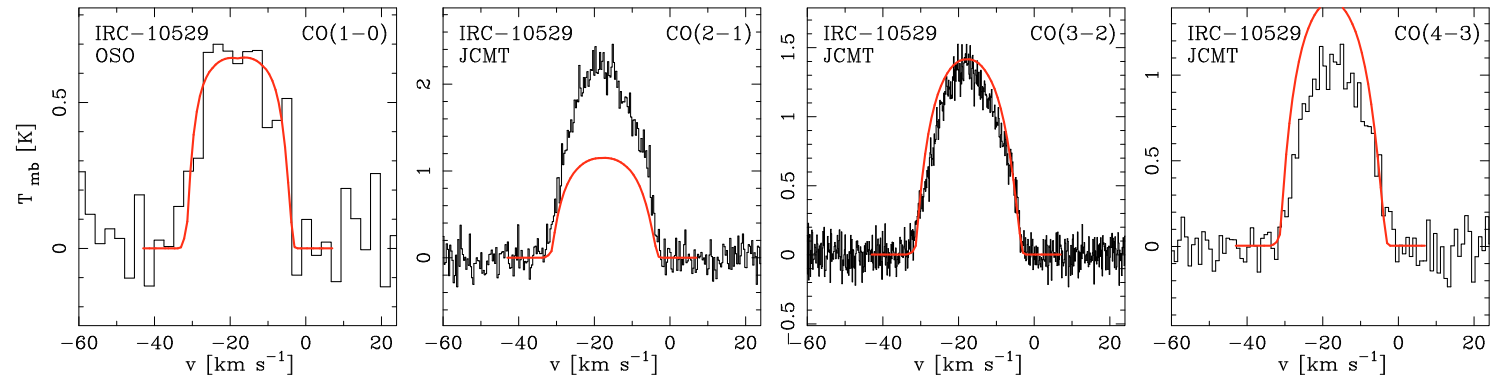

Fig. C.8. The observed CO $J=1 \rightarrow 0$ line from OSO, $2 \rightarrow 1,3 \rightarrow 2$, and $4 \rightarrow 3$ lines from JCMT, of the M-type star IRC-10529, overlayed with the best-fit model $\left(\dot{M}=1.6 \times 10^{-5} M_{\odot} \mathrm{yr}^{-1}\right)$ from the CO radiative transfer modelling. The reduced $\chi^{2}$ for this model is $\chi_{\text {red }}^{2}=4.6$. See Sect. 8.2.5. 